\title{
Rotational Invariants and Dynamic Touch
}

\author{
CLAUDIA CARELLO AND M. T. TURVEY
}

\begin{abstract}
Skilled users of a long-cane are quite fluid in registering the layout of surfaces that surround them. What they need to know, of course, concerns properties with consequences for behavior-whether the ground is sufficiently flat and solid to be negotiated safely, whether discontinuities in that ground surface are gaps that can be stepped over or brinks that must be descended. At first blush, this skill may seem like a remarkable cognitive achievement, one in which the unsighted individual has managed to associate tactile impressions with some basic spatial elements, perhaps plugging them into modified variants of the inferential algorithms presumed to serve vision. Such a characterization, however, ignores the fundamental informativeness of the haptic perceptual system in its own right. The skillful use of a long-cane is possible because the properties registered through touch are lawful and reliable. Wielding the cane and using it to strike and probe surfaces deforms the tissues of the hand, arm, and body. Despite moment to moment, incidental variation in the impressions on the skin, we argue that there must be reliable structure in the deforming tissue that informs about not only the probed surfaces but about the probe itself. The commonality between perceiving probes and perceiving by means of probes - more generally, between perceiving objects and perceiving by means of objects-reinforces the anchoring of what may seem like a highly specialized, extraordinary skill in the very ordinary operation of the basic haptic perceptual system.

Our goal in the present chapter is two-fold. First, we hope to highlight the integral contribution of haptics to the abilities not only of the blind but also of the sighted in getting around in and knowing about their surroundings. Second, we will make a case for understanding those abilities as fundamentally perceptual rather than inferential, an understanding that rests on an appreciation of the physical underpinnings of wielding hand-held objects.
\end{abstract}

\section{Haptic achievements escape everyday notice}

People generally have little difficulty imagining what it would be like to lose the sense of sight. Indeed, this loss is easily simulated simply by closing one's eyes. Similarly, people have little difficulty imagining what it would be like to lose the sense of hearing. Its loss is only marginally more difficult to simulate, requiring ear plugs or industrial grade ear protectors. Although these exercises are more intellectual than empathic, they are at least accessible to our understanding. But what would it be like to lose the sense of touch? What consequences could we imagine for our everyday lives? It is, perhaps, telling, that this musing is not common. Touch is not a sense that we think about having, let alone losing. In any event, we might begin with a surmise about the loss of sensitivity in the skin, but with what deprivation? One might fail to distinguish silk from wool or to notice the attack of a mosquito. The inadequacy of this initial conjecture, however, is suggested by conditions of temporary haptic loss, conditions which are not so much simulations as intrusive neurological events. For example, when the circulation is cut off in the lower leg (when the leg "falls asleep") it is not only things touching the skin that cannot be felt, it is the leg itself that cannot be felt as well as the floor it stands on. A shot of Novocain for a dental procedure reveals an additional consequence of losing feeling: the inability to position or control the insensate part.

These partial haptic losses hint at the severe consequences of a specific type of damage to the peripheral nerves, that affecting sensory fibers only. In contrast to blindness and deafness, which are not unusual genetic or adventitious conditions, complete sensory neuropathy is quite rare, numbering fewer than 10 cases in the medical literature. The most thoroughly documented case 
(Cole, 1995) is also one of the most severe: Ian Waterman lost all sensation below the neck at the age of 19.1 He has no awareness of where his arms, legs, and body are in space unless he is looking at them. More technically, he has no haptic proprioception. The consequences are dramatic (and would have been devastating had he not shown monumental determination to find ways to overcome them). Walking, standing, even sitting are under strict visual, exhaustively conscious control. He cannot walk in a crowd or on uneven terrain because he is unable to make the on-line adjustments needed to remain upright in response to a jostle or an awkward footfall. Indeed, he cannot make the necessary adjustments to remain standing should he sneeze or simply if the lights go out; he immediately falls to a heap on the floor. Actual walking is quite deliberate: He leans forward and downward to keep his legs and body in view, swinging each leg stiffly from the hip, slapping each foot down as a whole, using an abnormally wide stance to enhance stability.

After nearly two decades of extraordinary effort, both mental and physical, Mr. Waterman has made a remarkable functional recovery. With no organic improvement, he has taught himself how to substitute optical information and conscious strategies for the absent haptic information. While he can accomplish many tasks, his methods and goals are quite different from ours. The effort to draw a picture, for example, is largely directed at his own body position and gripping the pencil rather than at the drawing itself. When lifting an object, he knows that he has used the appropriate force depending on whether he sees the object come towards him or he sees himself move towards it (that is, if he sees himself falling over, he knows that the object is heavier than he had expected). Having learned to use vision to control walking, something as seemingly innocuous as a windy day alters - with dangerous variation and unpredictability - what he has learned about his usual postural relation to the visual horizon. He cannot walk quickly because there is a limit to how fast he can see the consequence of a step and make the next command. And because maintaining posture and walking are now mentally demanding, he cannot simultaneously do tasks that are themselves intellectual (e.g., taking notes without falling out of his chair) or delicate (e.g., holding an egg without crushing it).

The rarity of his condition meant that his doctors and therapists did not know how to treat it. ${ }^{2}$ But even more frustrating for Mr. Waterman was getting people to appreciate what was wrong: "I have lost a sense that few people know they have" (Cole, 1995). The pervasiveness of the consequences of his condition indicates how integral this unknown sense is to virtually everything we do. Feeling where the end of an object is or how heavy or wide it is allows us to do all of the mundane and indispensable acts of manipulation and transport: carrying an object; using it to poke or hit or scrape something; placing it on a table, on our head, in someone's hand. Feeling where the limbs are relative to each other, relative to the body, and relative to the world allows us to sit, stand, walk, reach, grasp, scratch an itch or comb our hair. Although our naïveté may consider touch only to be a skin sense - and, to be sure, Mr. Waterman has lost this sensitivity as well-the debilitation of neuropathy is dominated by the loss of what has been termed by Bell (1826) the muscular sense and by Gibson (1966/1983) effortful or dynamic touch. In this subsystem of the haptic system, muscular effort is used to move, say, a massive leg about a hip or a massive suitcase about the wrist and elbow. Mr. Waterman can perform such movements because he is not paralyzed. But his deficit is in controlling them, in feeling where the leg or suitcase is as it moves.

The touch system is unique among the senses in being both exploratory and performatory, both sensory and motor. As the case of Ian Waterman brings into bold relief, these are not separable functions. They are a compelling example of a perceiving-acting cycle (Kugler \& Turvey, 1987; Shaw \& Kinsella-Shaw, 1988; Turvey, Carello, \& Kim, 1990): Performing an act requires exploring objects and limbs and the self, perceiving their dimensions and their orientations relative to each other, allowing the appropriate forces and directions of forces to be used to move the objects and limbs where they're supposed to be, to and from positions and orientations that are continuously perceptible, continuously felt. The next section examines what might provide the basis for haptically perceiving these properties without benefit of vision. 


\section{Rotational dynamics and perceiving by dynamic touch}

Consider the method that Mr. Waterman developed for walking, swinging a leg stiffly from the hip. He freezes the ankle and knee, limiting the movement of his leg mass to rotation about a single joint. This contrived style of locomotion exaggerates a crucial fact: The movement of any limb segment can be characterized as a rotation about a joint. ${ }^{3}$ With or without haptic sensitivity, the body is constructed such that limbs move in rotary motions centered around the joints (Figure 1). Formally, such motions are describable by the physics of rotations or rotational dynamics. In the present example, the hip is the center of rotation and the ease of movement is determined by how much mass is being moved at how great a distance from that rotation point. Physics allows us a short-hand for locating the mass of the lumpy biological form that is a leg in terms of its center of mass, or $\underline{\mathrm{CM}}$. Adding a heavy boot to the foot would move the $\underline{\mathrm{CM}}$ in the direction of the foot. The consequence of this addition for rotating the leg is straightforward: The booted leg is harder to swing than is the unbooted leg. The booted leg has a larger resistance to rotation. ${ }^{4}$

Any of the various limb segments will resist being rotated as a function of how massive they are and how far that mass is from the axis of rotation. As it happens, the quantification of these resistances provided by rotational dynamics is quite informative about the dimensions and orientations of the limbs. And as the booted foot example begins to suggest, this story generalizes quite straightforwardly to objects attached to the body, including those tools and implements typically grasped in the hand. Let's turn our attention to a more formal treatment of rotational dynamics in order to appreciate how this can be so.

We begin by considering an L-shaped object such as a leg being swung about the hip, or a hand-held hammer being wielded about the wrist (Figure 2). A set of $\underline{x}^{-}, \underline{y}^{-}$, and $\underline{z}^{-}$axes can be anchored in those respective rotation points, permitting the calculation of resistances to rotation about each axis. Two things are immediately apparent: The distance of the $\underline{\mathrm{CM}}$ from the rotation point differs for the different axes, and the distribution of mass about each axis is not necessarily symmetrical. The implication of the first observation is that the resistance to rotation will depend on the

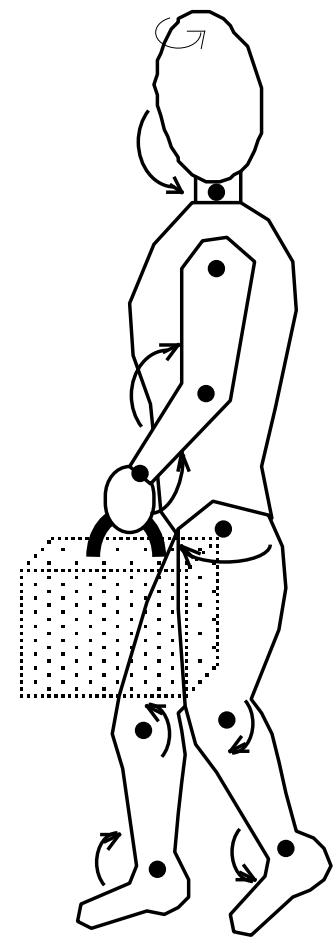

Figure 1. As a person walks, limb segments rotate about joints in differing amounts and in different directions. Even an apparently translatory motion such as lifting a suitcase is accomplished through rotary motions. 
(a)

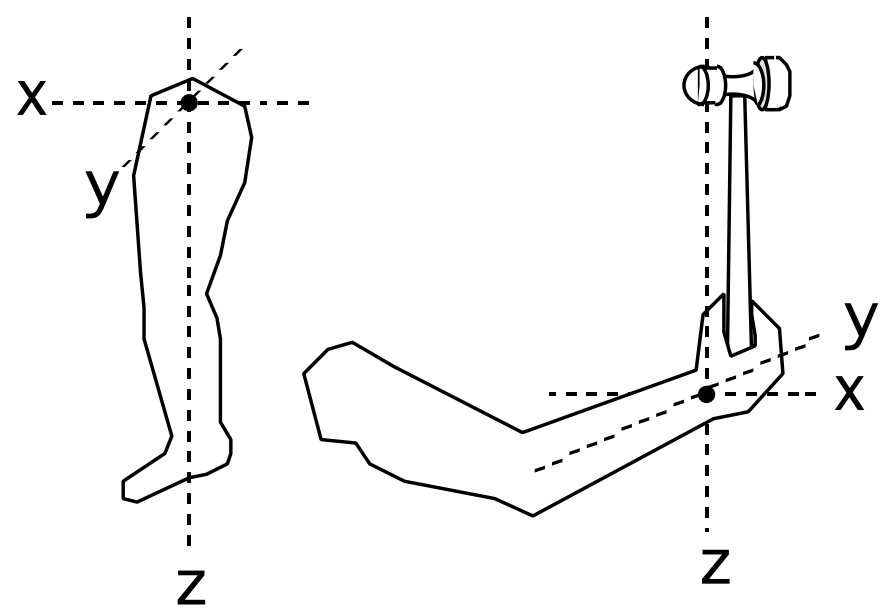

(c)

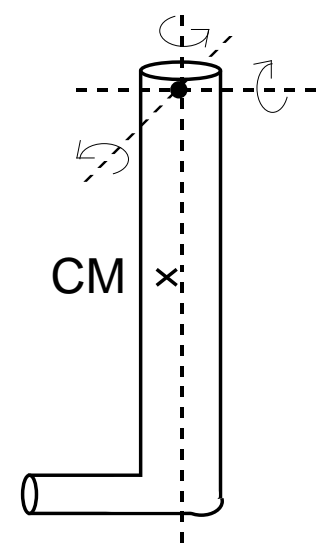

(d)

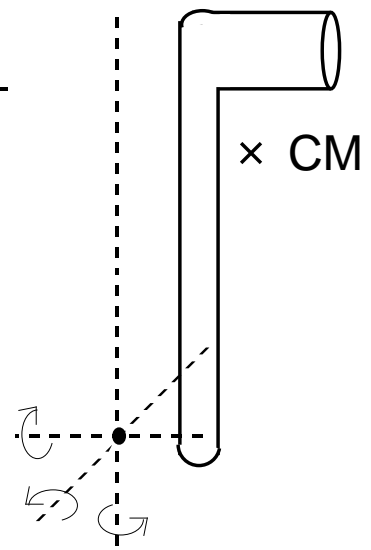

Figure 2. (a) The leg swings forward and back about the $\underline{x}$-axis, side to side about the $\underline{y}$-axis, and twists about the $\underline{z}-$ axis. (b) An upholsterer's hammer grasped firmly in the hand swings up and down about the $\underline{x}$-axis, side to side about the $y$-axis, and twists about the z-axis. (c) The resistances to rotation about the axes defined for the leg are calculated based on the distance of the $\underline{\mathrm{CM}}$ from each of them. (d) The resistances to rotation about the axes defined for the hammer are calculated based on the distance of the $\underline{\mathrm{CM}}$ from each of them.

axis of rotation. In the depicted examples, the z-axis has been chosen to be parallel with the longitudinal axis of the objects. Since, the CM is closest to this axis, the resistance to the twisting motion about $\underline{z}$ will be quite a bit smaller than the resistance to the swinging motions about the $\underline{x}$ - and $\mathrm{y}$-axes, which themselves will be roughly comparable. The implication of the second observation is that there will be resistances in directions off the axial rotations. In effect, there will be a tug in the direction of the asymmetry.

What we have described in the foregoing are the moments and products of inertia of an object with respect to an axis of rotation, which can be captured in a $3 \times 3$ symmetric matrix called the inertia tensor (Figure 3a). Note that this particular inertia tensor is defined with respect to a particular set of axes. For example, although we elected to run the z-axis parallel with the longitudinal axis of the objects, this election - though intuitively appealing-is mathematically arbitrary. Indeed, any other set of orthogonal axes through the point of rotation would be equally legitimate (or equally arbitrary). Any other set, that is, save one: The symmetry axes of the object, the axes about which

(a)

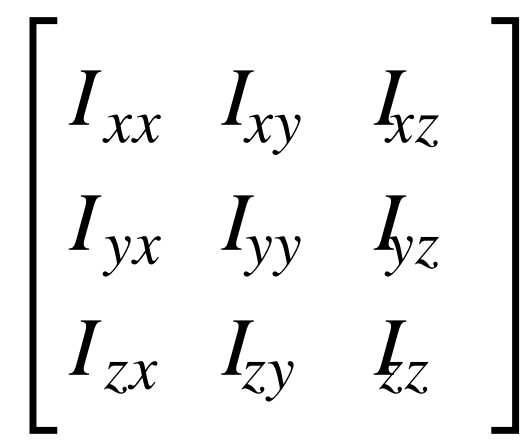

(b)

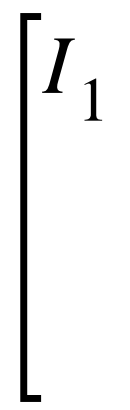

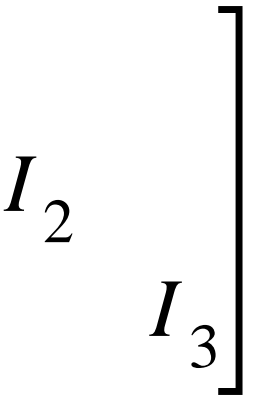

Figure 3. (a) The inertia tensor is a $3 \times 3$ symmetric matrix with moments of inertia on the diagonal and products of inertia off the diagonal (the values above the diagonal are the same as those below the diagonal). It quantifies different resistances to rotation in different directions. (b) When the tensor is diagonalized, the products of inertia go to zero leaving only the principal moments of inertia. 
the mass distribution is even relative to the rotation point, constitute the only nonarbitrary set. Fortunately, and powerfully, they can be derived from any inertia tensor of the preceding kind by diagonalizing that tensor, in effect, reorienting the axes so as to eliminate the off-diagonal components. The symmetry axes are called eigenvectors or principal directions $\underline{e}_{k}$ and their lengths are given by eigenvalues or the principal moments of inertia, $\underline{\mathrm{I}}_{\underline{k}}$ (Figure $3 \mathrm{~b}$ ).

This description provides a substantial portion of what is needed to characterize the sizes, shapes, and orientations of objects. In particular, it allows us to construct a geometric representation of an object, its inertia ellipsoid, that entails the essence of its mass distribution (Figure 4). The eigenvalues of the ellipsoid map into the magnitudes of objects whereas the eigenvectors of the ellipsoid map into the directions of objects. The depicted ellipsoid is elongated and tilted, for example, reflecting the fact that both the leg and the hammer are rotated from one end and have an attachment of sorts going off in one direction. The more evenly distributed the mass is about the center of rotation, and the closer that point is to the object's $\underline{\mathrm{CM}}$, the more spherical and less tilted will be the ellipsoid.

The inertia tensor is a physical invariant. Given the receptive machinery that is embedded in the muscles and tendons, it may well be just the physical invariant that that machinery is suited to extracting from interactions with the physical world. Muscle spindles and Golgi organs respond collectively to muscle deformation, that is, their response is field-like. In providing quantifications of strain differences in different directions, stress differences in different directions, rates of change of strain differences in different directions and, potentially, rates of change of stress differences in different directions, their activity seems to provide the basis for a tensorial treatment of tissue states. ${ }^{5}$ As would be expected from this account, sensitivity by the muscle spindles and Golgi organs is missing in Ian Waterman.

Over thirty years ago, Gibson (1966/1983) asserted that "the stimulus information from wielding can only be an invariant of the changing flux of stimulation of the muscles and tendons" (p. 127). Following ideas first advanced by Solomon (1988; Solomon \& Turvey, 1988) we have elaborated this position to focus on the invariant inertia tensor as a quantification of object and body properties that might generate reliable structure in deforming tissue that informs about those properties (Turvey \& Carello, 1995a; Turvey, 1996). The tensorial model of dynamic touch provides a straightforward logic for experimentation. The next section lays out that logic and then summarizes research that has been conducted to test it.

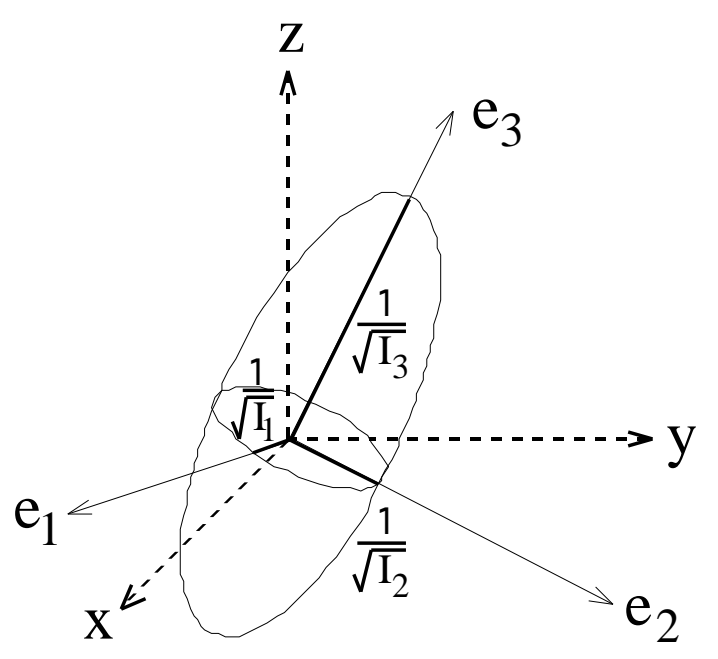

Figure 4. The inertia ellipsoid is oriented with respect to the symmetry axes or eigenvectors, $\underline{\mathrm{e}}$, of the object relative to the rotation point. Its size - that is, the lengths of the eigenvectors-is determined by the principal moments or eigenvalues, $\underline{I}_{\mathrm{k}}$. 


\section{A framework for studying dynamic touch}

The tensorial account of dynamic touch has developed within James Gibson's ecological approach to perception (Gibson, 1979). This approach is organized around the central theme that perception is lawful (Turvey \& Carello, 1981; Turvey et al., 1990; Turvey, Shaw, Reed, \& Mace, 1981; Turvey \& Shaw, 1995). An ecological law takes the following form:

$$
\begin{aligned}
& \text { animal-environment } \\
& \text { relation }
\end{aligned}
$$

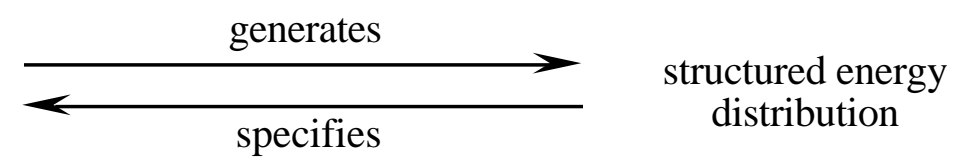

In this formulation, lawfully structured energy distributions can include light, sound pressure, tissue deformation, and chemical diffusion (and, for animals other than humans, ultraviolet radiation, magnetic fields, etc.). Insofar as the structured energy is lawfully related to the circumstances that gave rise to it, it is informative about those circumstances. The tensorial account holds that, for dynamic touch, the lawfulness of tissue deformation is to be found in the quantifications provided by rotational dynamics. Bluntly, to the extent that a property affects the tensor (i.e., is reflected in the tensor) it should affect perception.

Consider a simple experiment on perceiving length by dynamic touch. A participant is asked to grasp an object at one end and wield it out of view behind an occluding screen. Magnitude production typically serves as an intuitive response measure in which the felt length of the wielded object is matched by positioning an adjustable visible marker (Figure 5a). In particular, participants are asked to adjust the position of a surface in front of them so that it could just be reached with the tip of the wielded object. When the objects are cylindrical rods of a homogeneous material, perceived length tracks actual length quite faithfully (Figure 5b). As the axis of rotation is changed, by changing the grasp from the end to the middle (Figure 5c) - thereby reducing the resistance to rotation-perceived length decreases (Solomon \& Turvey, 1988, Experiment 7). A comparable effect is obtained by attaching a mass to the rod at various positions (Figure 5d). Namely, perceived length increases the further the mass is from the axis of rotation in the wrist, that is, the greater the resistance to rotation (Solomon \& Turvey, 1988, Experiment 5).

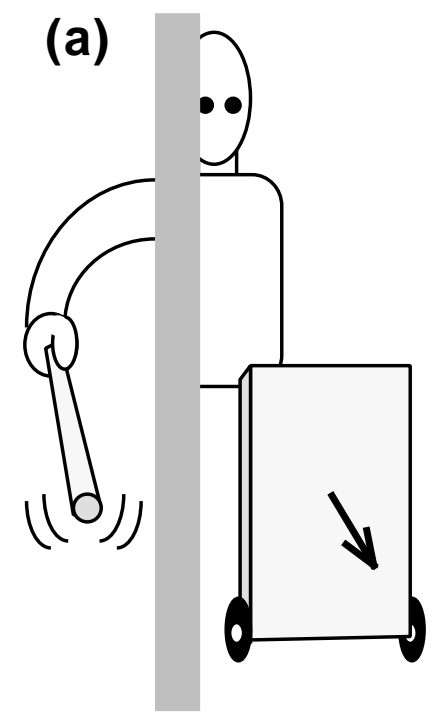

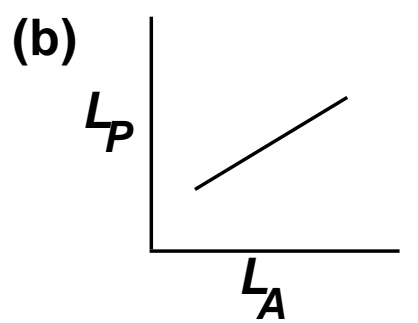

(c)

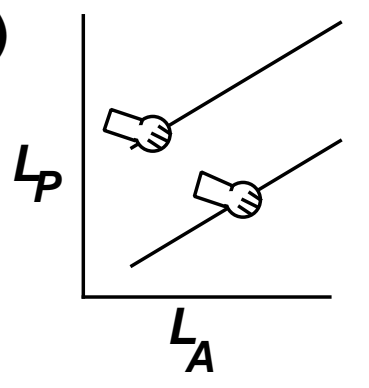

(d)

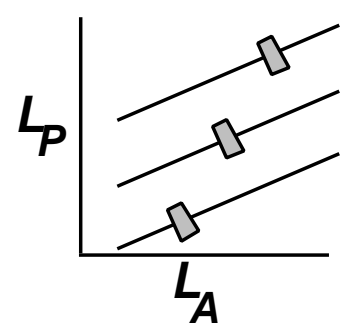

(e)

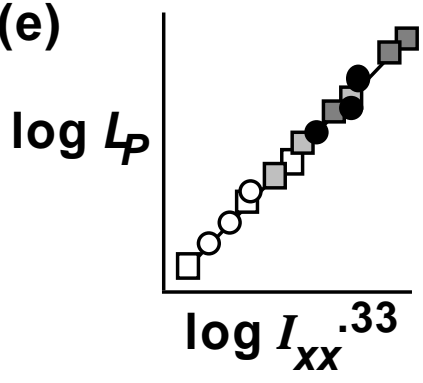

Figure 5. (a) An object is grasped firmly and wielded on one side of curtain while a visible report board is adjusted to coincide with the felt location of the tip of the rod. (b) Perceived length $\underline{L}_{\mathrm{P}}$ of homogenenous rods is in the range of actual length $\underline{L}_{A}$. (c) $\underline{L}_{P}$ increases as the grip is moved farther from the rod's $\underline{C M}$. (d) $\underline{L}_{P}$ increases as an attached mass is moved farther from the axis of rotation in the wrist. (e) $\underline{L} \underline{P}$ in the foregoing is a function of the rod's maximum moment of inertia raised to the $1 / 3$ power (in $\log$ - $\log$ coordinates). 
Several programmatic issues are illustrated by these simple examples. Note that a given actual rod length is associated with more than one perceived length. Under a traditional analysis, one might be tempted to label these instances of haptic length perception illusory since they are modulated by manipulations of variables other than length. But the ecological law framework is an argument for the specificity of perception to information. This means that, under the appropriate analysis, the perception $\rightarrow$ information mapping must be one-to-one. To the extent that the mapping appears to be many-to-one, it is an indication that the analysis is not appropriate- the scientist has not yet identified the informational support for that particular perception. A many-to-one map is likely if one assumes that the physical or geometric properties that are easily labeled are necessarily the properties that perceivers should be sensitive to. In a very real sense, therefore, we would say that the scientist, not the perceiver, is in error $^{6}$ (Michaels, Carello, \& Shapiro, 1985). In contrast, the search for single-valued functions that is entailed by ecological realism serves as a useful "engine of discovery" in research (Turvey \& Carello, 1995b), as will be seen with the next example.

We have to be careful in these kinds of summaries to keep in mind that the framework described in the preceding section is the result of over 10 years of theoretical and empirical development. Our earliest research was only beginning to explore the possibility that rotational inertia was the major constraint on perceiving object properties by dynamic touch. Consequently, we imposed certain methodological limitations in order to keep the calculations as simple as possible. Even though Solomon and Turvey (1988) recognized the relevance of a tensorial description-that different resistances in different directions should be captured by a hypernumber, that diagonalizing a tensor would provide the only set of nonarbitrary axes-experiments focused on one (undiagonalized) tensor component at a time. The objects, for example, tended to be thin rods of a single material. For such objects of cylindrical symmetry, the resistances to rotation about the $\underline{x}-$ and $\mathrm{y}$-axes are equivalent; given $\underline{\mathrm{I}}_{\mathrm{xx}}, \underline{\mathrm{I}}_{\mathrm{yy}}$ is superfluous. Moreover, $\underline{\mathrm{I}}_{\mathrm{zz}}$, the resistance to rotation about the z-axis, was not considered because diameter was treated as negligible. Under these circumstances, the basic equation for moment of inertia for a cylinder about its $\underline{\mathrm{CM}}$ simply reduces to $\underline{\mathrm{ml}}^{2} / 12$. But diverse circumstances soon complicate matters. For example, when the axis of rotation is somewhere other than at the $\underline{\mathrm{CM}}$, moment of inertia is greater. The amount of increase is dictated by the Parallel Axis Theorem, which essentially increments the basic equation with a term that reflects the resistance to rotation about this new axis (an axis parallel to one through the CM). It is given by the product of the rod mass and the squared distance of the $\underline{\mathrm{CM}}$ from the actual axis of rotation. Note that the parallel axis component is required even in the case of a rod grasped at its $\underline{\mathrm{CM}}$ because the rotation point is in the wrist, off the central axis of the rod.

The characterization restricted to $\underline{I}_{\mathrm{xx}}$ works perfectly well when the mass distribution is manipulated through a change in grip or rod length or even the addition of small mass somewhere along the rod's length (Figure 5e). But the equation makes it obvious that the heavier the rod, the longer it should feel. For a given length, rods can be made heavier by choosing them to be of larger diameters or denser materials. If $\underline{I}_{\mathrm{xx}}$ is the only constraint on perceived length, denser or wider rods should be dramatically overestimated relative to lighter, thinner rods. Indeed, experiments that include rods of different diameters or different densities or different shapes (Figure 6) reveal that the heavier or wider ones are perceived to be longer (Fitzpatrick, Carello, \& Turvey, 1994). But they no not feel as long as $\underline{I}_{\underline{x x}}$ alone would suggest. More importantly, at least for purposes of

(a)

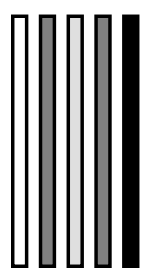

(b)

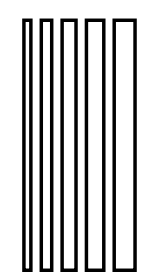

(c)

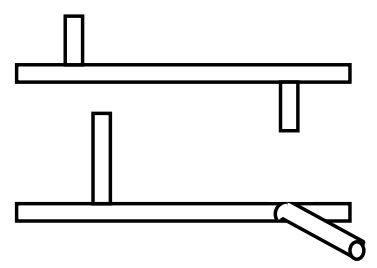

(d)

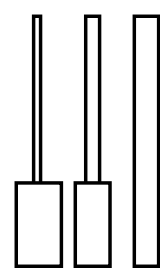

(e)

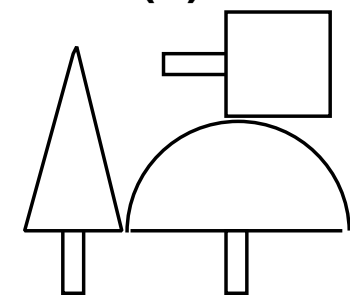

Figure 6. Objects in length perception experiments can vary in (a) material density, (b) diameter, (c) symmetry, (d) homogeneity, or (e) shape. (Objects of type (c) are from Pagano \& Turvey, 1992; the remainder are from Fitzpatrick et al., 1994). 

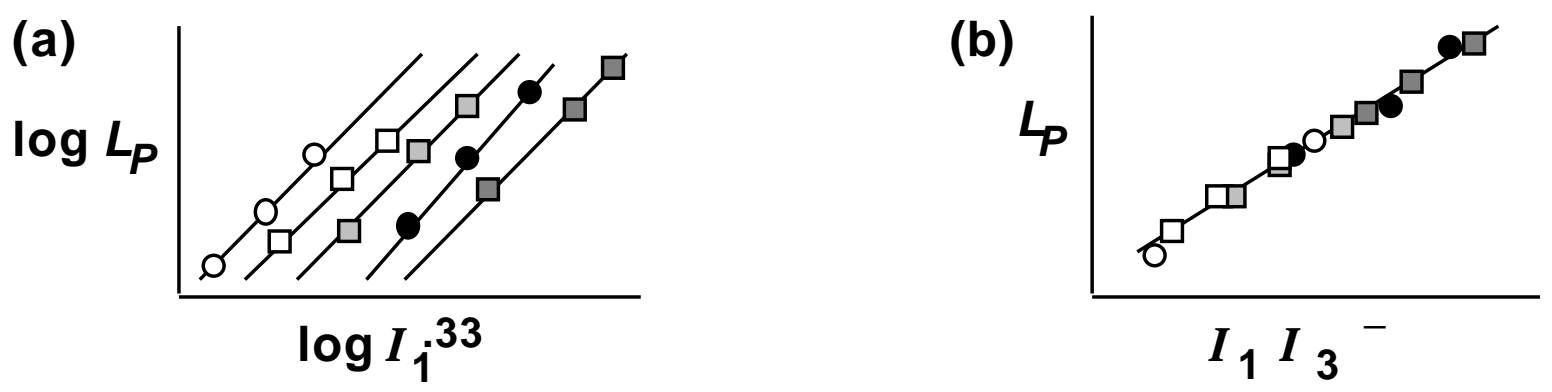

Figure 7. (a) Rods of different diameters produce functions of $I_{1}$ with different intercepts. (b) When the full tensor is considered, all of those object are accommodated by a single function (Fitzpatrick et al., 1994).

illustrating a scientific strategy, regressions of perceived length on $\underline{I}_{x x}$ produce many-to-one functions (Figure 7a). This fact must be taken as evidence that our limited characterization of $\underline{I}_{\mathrm{xx}}$ as the physical constraint on perceiving length was inadequate. Abandoning the simplifying assumption of negligible diameter most obviously implicates $\underline{I}_{z z}$. This, in turn, brings the realization that the whole tensor should be considered, most generally in diagonalized form (see Figure 3 ). Now a multiple regression of perceived length on the diagonalized tensor (in log-log coordinates) reveals a significant dependence on both the maximum eigenvalue $\underline{I}_{1}$ and the minimum eigenvalue $\underline{I}_{3}$. The regression equation so obtained can be used to generate a power function to characterize the entire data set. Essentially, the coefficient of $\log \underline{I}_{1}$ becomes the exponent for $\underline{I}_{1}$ and the coefficient of $\log \underline{I}_{3}$ becomes the exponent for $\underline{I}_{3}$. The resulting power function-in which $\underline{I}_{1}$ is raised to a positive power and $\underline{I}_{3}$ is raised to a negative power-is used to generate predicted values of perceived length. In allowing a simple regression of perceived length on the power functiongenerated values, this technique highlights the fact that a single function fits perception of objects that vary widely (Figure 7b). Despite variations in density (wood, aluminum, and steel), diameter $(.25$ to $.64 \mathrm{~cm})$, shape (cubes, cones, cylinders, pyramids, and hemispheres all grasped by the same handle), and homogeneity (thick wooden handles with thinner steel rods; thick wooden handles with thick wooden rods), the scaling is the same. Speaking practically, the negative exponent on $\underline{I}_{3}$ serves to moderate the contribution of increased mass per unit length, effectively keeping perceived lengths of the variety of objects within the appropriate range. This, of course, is our experience in using objects-our ability to control wooden pencils, for example, does not seem to be appreciably different from our ability to control metal or plastic pens.

We should emphasize some of the methodological aspects of the experiments we are describing here. Participants have no idea about the number of objects, their lengths, materials, or shapes. They are given no feedback about their responses. And the response apparatus allows length reports anywhere from 0 to $2 \mathrm{~m}$. Nonetheless, participants use a limited part of the apparatus, a range corresponding roughly to the range of actual lengths. They do not simply distinguish lengths on a relative scale (in which case they could just as likely use the whole apparatus or a small part of it, with the actual magnitude arbitrarily elected; Gogel, 1977) nor do they provide perceived lengths that scale absolutely to actual lengths (the inertial constraint means that they cannot). Instead, they provide what appears to be a definite scale (Bingham, 1993; Carello, Fitzpatrick, Flascher, \& Turvey, 1998; Peck, Jeffers, Carello, \& Turvey, 1996). What individual differences exist in perceived length are, from our perspective, nothing to get exercised about because every participant is operating within essentially the same scale. Not only are their individual regressions similar but, more dramatically, if different participants each contribute one mean for randomly selected rod configurations (i.e., the mean for Rod 1 comes from Participant 5, Rod 2 from Participant 6, etc.; cf. Bingham, 1993), each "random pairing regression" is significant and shows the $1 / 3$ scaling for homogeneous rods (Peck et al., 1996).

The notion of definite scaling brings us back to the issue of whether these perceived lengths should be considered illusory simply because they are not metrically faithful. Philosophical considerations aside (see Footnote 6), the systematicity of performance, as well as its anchoring in the mechanics of people's interactions with these objects, argue against such an interpretation. Metrical 
departures coupled with apparent lawfulness raise the question of whether it is even legitimate to consider length to be the property that is being perceived. To be sure, participants would have no difficulty in performing the task if it were described as "use this visible surface to indicate how long the wielded rod is." But we put the instructions in functional terms for a reason. In Gibson's ecological approach to perception, animals are said to perceive affordances, the behavioral possibilities of an environmental layout for that animal (Gibson, 1979). Recall that we opened this chapter with a description of what users of a long-cane need to know, for example, whether the ground surface supports normal locomotion. It is our contention that an affordance description will provide the ultimate understanding of what wielding tells us about objects. At the heart of what the mass distribution captures about an object is the ways that the object can be used. Those uses are not simply a function of geometric dimensions such as length and width but, in a very real sense, how those objects can be moved (e.g., whether or not they are "unwieldy").

Developing a principled understanding of appropriate functional properties remains a deep challenge that we begin to address later in this chapter (in a section on affordance and the perception of heaviness). Getting to the point where such a treatment is conceivable built upon work in which we unashamedly continued to ask wielders about length-like and width-like properties. Although not explicitly functional, the work to which we now turn reveals both the richness of haptic awareness and the physical constraints on that awareness that form the principled basis for the more functional account.

\section{Eigenvalues specify magnitudes; eigenvectors specify directions}

Contemporaneous with the experiments on object length were experiments on object orientation. These, too, took the approach of focusing on individual tensor components. As noted earlier, the off-diagonal terms or products of inertia in some sense reflect the asymmetry of the mass distribution of a hand-held object. To the extent that an asymmetric object can be said to have an orientation, it was expected that the products of inertia would constrain perception of that orientation. This was found to be the case using a simple variation on the hand-held rod, namely, the hand-held L-shaped rod (Turvey, Burton, Pagano, Solomon, \& Runeson, 1992). The question was simply, "which direction is the branch pointing?" Responses were provided by adjusting a pointer on a dial to coincide with the orientation of the branch; performance indicated that object orientation could be perceived by dynamic touch (Figure 8). In order to verify that the physical constraint was actually provided by the inertia tensor and not simply spatial orientation (whether or not the latter could have any meaning apart from the tensor), special rods were constructed. These had Vbranches in which one of the arms of the V could be weighted (Pagano \& Turvey, 1992). Whereas the eigenvector orientation is affected by whether the left or right branch is weighted, the spatial
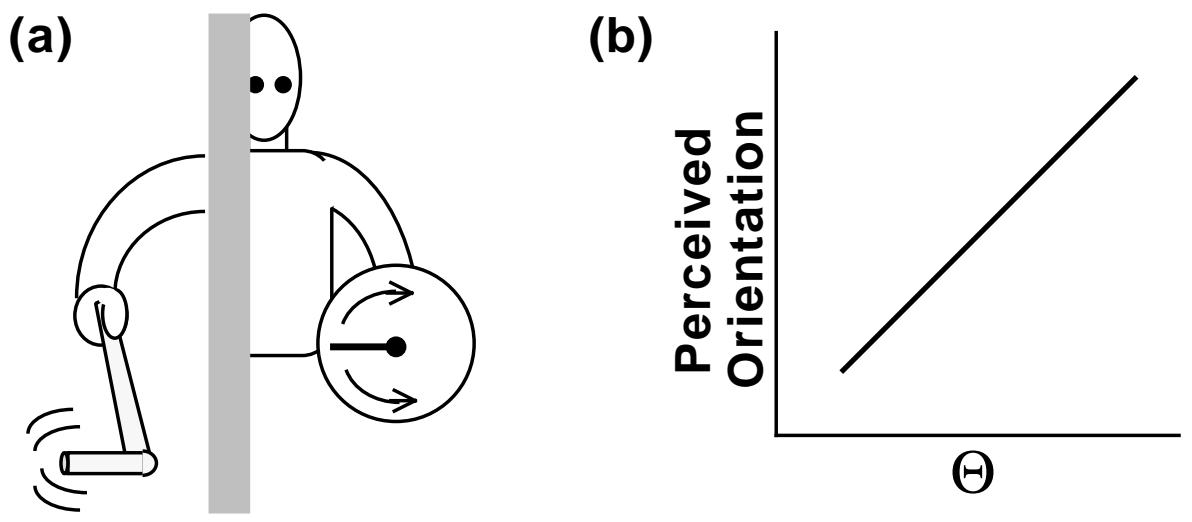

Figure 8. (a) An occluded L-shaped rod is wielded as the orientation of its branch is indicated with a dial.

(b) Mean perceived orientation tracks actual orientation. 

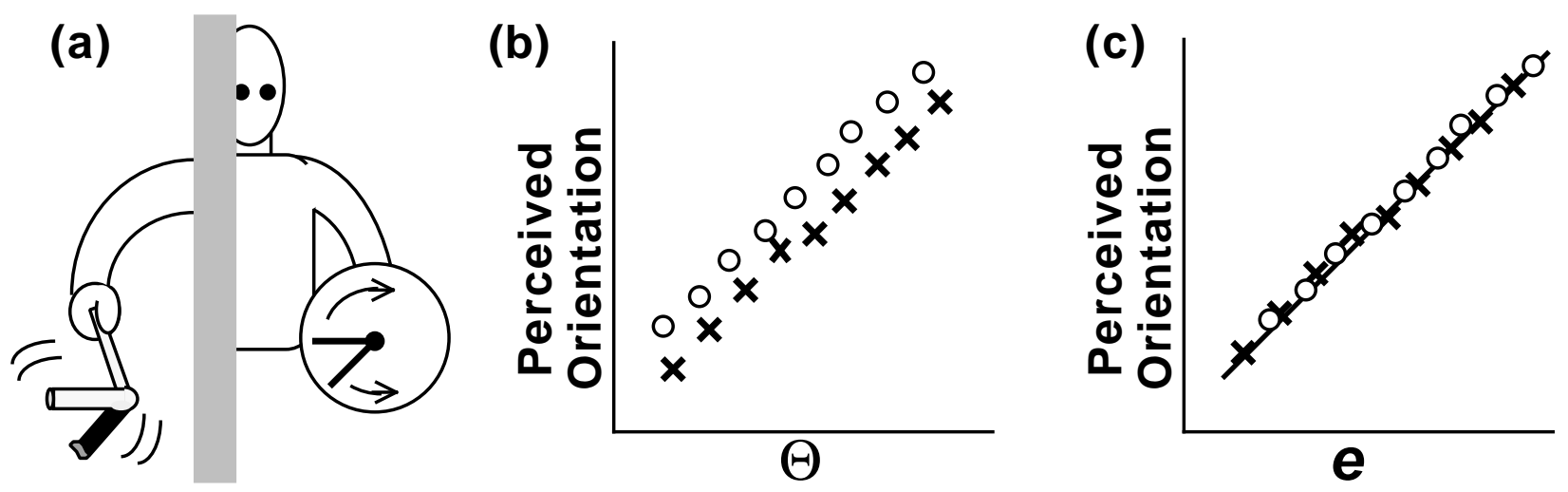

Figure 9. (a) One branch of a $\mathrm{V}$ is weighted to divert the orientation of the eigenvector from the spatial orientation of the object. A dial is used to report the orientation of both branches or just the weighted one. (b) Perceived orientation departs from spatial orientation, but (c) tracks eigenvector orientation.

orientation of the branches is not. Once again, responses were provided by adjusting a dial, either a single pointer to indicate the orientation of the weighted branch or two pointers, one for each branch. And once again, the tensorial hypothesis was supported (Figure 9).

The two preceding lines of research, one concerned with perception of length and one concerned with perception of orientation, are at the core of much of our current understanding of perception of object properties by dynamic touch. This understanding makes explicit the parallels between how object mass distributions structure the tensor and how the tensor, in turns, specifies object properties. Recall that the mass distribution of an object is captured by the orientation, size, and shape of the inertia ellipsoid, which is itself given by the orientations of the eigenvectors as well as their lengths or eigenvalues. What the summarized research has shown is that perceived magnitudes (such as length) are single-valued functions of the eigenvalues and perceived directions (such as object orientation) are single-valued functions of the directions of the eigenvectors.

More recent research builds on this theme. Other perceived magnitudes such as width and height (Turvey, Burton, Amazeen, Butwill, \& Carello, 1998) or weight (Amazeen, in press; Amazeen \& Turvey, 1996) are constrained by eigenvalues as expected. Other perceived directions such as where the hand is on an object are constrained by the directions of the eigenvectors as expected (Pagano, Carello, \& Turvey, 1996; Pagano, Kinsella-Shaw, Cassidy, \& Turvey, 1994). Note that any given object grasped firmly in the hand will have only one tensor. Nonetheless, that tensor supports the perception of a variety of properties. When the property to be perceived differs, the parsing of the tensor differs. For example, object height and object width are easily distinguished perceptually, and their tensorial dependencies are also distinct: The positive exponent on $\underline{I}_{1}$ is larger for height than for width; the exponent on $\underline{I}_{3}$ is negative for height and positive for width (Turvey et al., 1998).

How, then, does one perceive through "the changing flux of stimulation of the muscles and tendons" what one needs to perceive? The issue being raised here is really a matter of selective perception or attention. This prominent topic of perceptual theories is not often considered in discussions of perception by touch but, as we shall see, this domain provides an interesting perspective on old questions.

\section{Selective perception by dynamic touch}

The discussion of attention is rooted in a more basic question: What determines whether a given property will be perceivable? We have offered the inertia tensor as the major constraint on perceiving object properties by dynamic touch. Properties unrelated to mass and its distribution (e.g., surface texture) obviously cannot influence the inertia tensor. But even properties that are 
related to mass and its distribution may fail to be distinct in the tensorial description and fail to be distinguished in perception. For example, even though shapes can be distinguished by dynamic touch, certain shapes are confused: cylinders with cubes and cones with pyramids (Burton, Turvey, \& Solomon, 1990). The confusions are understandable in tensorial terms: Confusable objects are closely related in their eigenvalues (in particular, in the ratio of $\underline{I}_{1}$ to $\underline{I}_{3}$ ). Angularity, the property that distinguishes these particular pairs of objects, is not captured in the tensor. This kind of fact suggests a candidate axiom of the tensorial account of perceiving by dynamic touch: To the extent that a property is not distinct in the rotational dynamics, that property should not be distinguishable by dynamic touch.

Having established the importance of the inertia tensor, however, we have provided ourselves with a puzzle: Given that there is only one inertia tensor for a given hand-rod configuration, what allows us to perceive more than one property, for example, both length and width? The general form of this problem is no more peculiar to touch than it is to seeing or hearing. The tensor is the inertial array that is informative about properties that structured it just as the optic array and the acoustic array are informative about the properties that structured them. While it is nontrivial to uncover what information supports the perception of length, width, weight, and so on, the strategy for doing so is more or less in place. But there is depth to this puzzle. Consider the situation in which a rod is held somewhere other than at the end. An individual asked to perceive just the portion in front of the hand can do so (Burton \& Turvey, 1991; Solomon, Turvey, \& Burton, 1989). This fact alone is not necessarily surprising and, moreover, the tensorial dependency on both a moment and a product of inertia follows from a characterization of partial length as a magnitude in a direction (Carello, Santana, \& Burton, 1996; Pagano et al., 1996). The puzzle arises because an individual can, in fact report the portion on either side of the hand and these perceptions are constrained by the same moment and product of inertia (Turvey, Park, Dumais, \& Carello, 1998). But as we have noted, the appearance of a 1:many map between a candidate physical variable and the perceived property it is thought to constrain demands more scrutiny.

For a single rod grasped in the middle (or two rods held in one hand; Turvey, Carello, Fitzpatrick, Pagano, \& Kadar, 1996) what distinguishes perception of one side from the other is the sign on the product of inertia. It is as if a perceiver elects to multiply the contribution of the product by -1 when attention is directed to one side of the grasp. Straining credulity, perhaps, it is as if all participants elect to multiply the contribution of the product of inertia by -1 when attention is directed to that same side of the grasp. While those with more cognitive tastes might be willing to accept that an algorithm, perhaps biased toward the thumb, could account for such universality in the application of a multiplicative rule, ecological psychologists are limited by another axiom of the tensorial account of perceiving by dynamic touch: To the extent that a property is perceivable by dynamic touch, its informational support must be distinct in the rotational dynamics.

Adhering to this axiom is not necessarily an easy route. Nearly a decade passed between the first experiments directed explicitly at the two-valuedness problem and our understanding of a physically tenable solution-and that understanding is mathematically dense (Turvey et al., 1996, 1998). For present purposes we note that the solution is rooted in the inertia ellipsoid characterization, in particular, that the orientation of the ellipsoid relative to the hand is brought about by a rotation. Intuitively, we can appreciate that a particular orientation or attitude of the ellipsoid can be brought about by, say, a small rotation in one direction or a larger rotation (through the complementary angle) about the same axis in the opposite direction (Figure 10a). These oriented rotations are at the heart of the solution to this problem. The attitude spinor (Hestenes, 1994a, b) effectively connects the rod's reference frame to the hand's. The spinor, $\underline{A}$, is the source of the multiplier -1 ; the contrast in attention (left versus right, above versus below) is equated with the selection of one of the two oriented rotations represented by A (Figure 10b). Hence, selective attention is itself rooted in rotational dynamics: Selective length perception by dynamic touch is a function of a quantity composed from $\underline{A}$ and $\underline{I}_{k}$ (Turvey et al., 1996). The spinor characterization thereby guarantees invariance in perception when there should be invariance (perception of a given portion of a rod does not differ as a function of its direction from the hand) and variance when there should be variance (perception of two different portions of a rod differ regardless of their respective directions). 

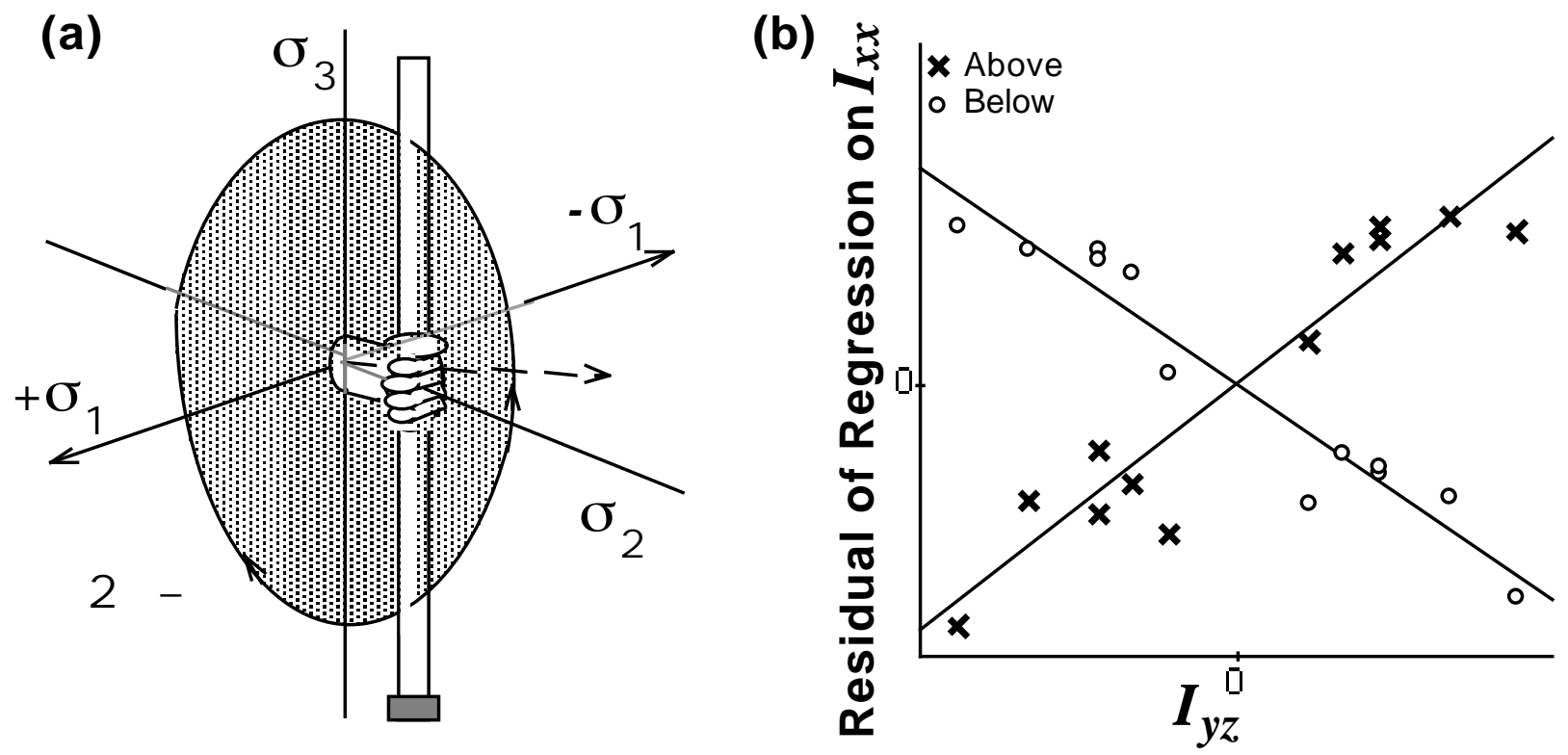

Figure 10. (a) The spinor is an oriented rotation that fixes the reference frame of the object relative to the hand. (b) It provides the change in sign of the product of inertia that distinguishes attention to two different directions.

Although we find the arguments for a physical basis to all phenomena of dynamic touch to be compelling, not everyone is so persuaded. Others embrace a perspective with a long tradition in psychology, the notion that one percept can be derived from another percept. Apart from logical persuasion, there are analytic methods adjudicate these positions and it is to them that we now turn.

\section{Percept-percept coupling vs. perceptual independence}

Helmholtz did much to direct the field toward an understanding of perception as the result of an unconscious inference. Oftentimes, that inference included the use of the perception of one property together with some knowledge in order to produce perception of another property. In the hallmark example, size perception is the result of the combination of perceived distance with knowledge of how distance and size go together when an image is projected on a surface. In such an instance of percept-percept coupling (Epstein, 1982), the perception of one property is mediated by the perception of another property. Many of the haptic perceptions that we have described have been considered by others to be candidates for perceptual dependence. For example, perceived partial length has been described as derived from perceived whole length and grip position (Chan, 1994); perceived whole length has been thought to depend on perceived weight (for wielding, Chan, 1995; for static holding, Lederman, Ganeshan, \& Ellis, 1996). For the most part, these kinds of conclusions have been based on correlations between two percepts. But as can be appreciated from preceding discussions of the physical constraints on perceived length, perceived partial length, and perceived weight, a correlation might be expected simply because all are functions, in varying combinations with other tensor quantities, of $\underline{I}_{1}$.

Epstein recognized that the conclusion of percept-percept coupling required proof beyond the simple observation that two percepts were correlated. He recommended that the coupling be evaluated statistically, by means of partial correlation (Epstein, 1977). The goal of such an analysis is twofold: to show that the relationship between the focal and the mediating percepts remains after their dependence on a physical variable has been removed and, relatedly, that the relationship between the focal percept and the physical variable declines when the dependence on the mediating percept has been removed. The analysis requires that, over the course of the experiment, both types 
(a)

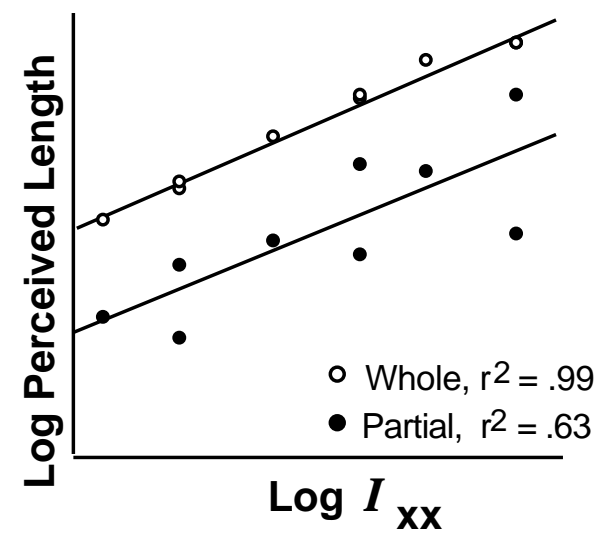

(b)

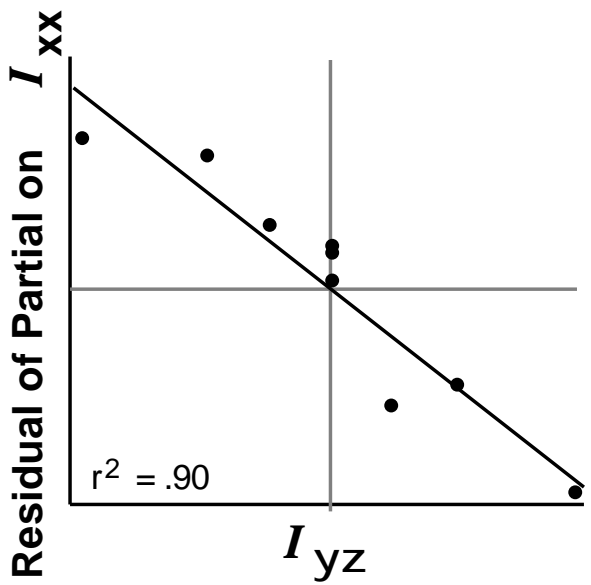

Figure 11. (a) Both perceived whole length and perceived partial length are functions of the maximum moment of inertia. (b) Only the regression of perceived partial length has a residual that is a significant function of the one nonzero product of inertia.

of responses be collected from each participant for every object. Perceived whole length and perceived partial length have been examined under such conditions and the conclusion of perceptpercept coupling was not supported (Carello et al., 1996). Consistent with the tensor hypothesis, these two properties were constrained by different physical quantities (Figure 11).

Although the preceding makes a case against the dependence of one percept on another, it has been argued that a more rigorous test is required. Just as correlation between responses need not mean dependence, the lack of correlation between responses does not necessarily mean perceptual independence (e.g., Ashby \& Townsend, 1986). The lack of correlation must characterize the entire distributions, not just the mean values of the responses. That is, true independence requires that perception of one dimension of a stimulus is unchanged across all levels of the other dimension of the same stimulus. A rigorous evaluation of independence is provided by the conjunction of an experimental design and an analytic procedure that allows all possible relationships among responses and stimulus dimensions to be encountered and measured. In the design for a complete identification experiment (Ashby \& Townsend, 1986; Maddox \& Ashby, 1996), a set of stimuli vary orthogonally on the relevant dimensions $\underline{A}$ and $\underline{B}$ (e.g., length and weight). Responses $\underline{a}$ and $\underline{b}$ (e.g., perceived length and perceived weight) to those two potentially independent dimensions are obtained for each stimulus. The key is that the manipulation and the responses are categorical (e.g., "short," "middle," and "long" versus "light," "medium," and "heavy"). In building orthogonality into the stimuli and providing the opportunity for it to be mirrored in the responses, the design has an advantage for showing independence in dynamic touch, an independence which is otherwise obscured because of the shared underlying tensorial constraint (Figure 12).

(a)

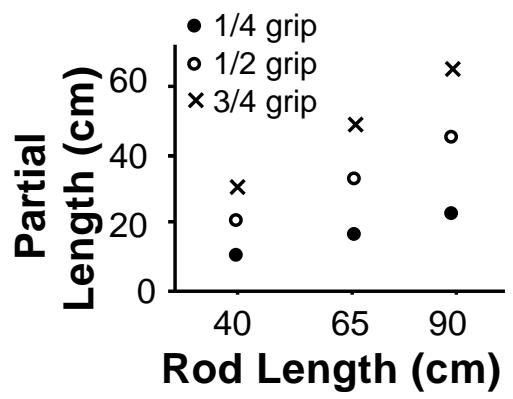

(b)

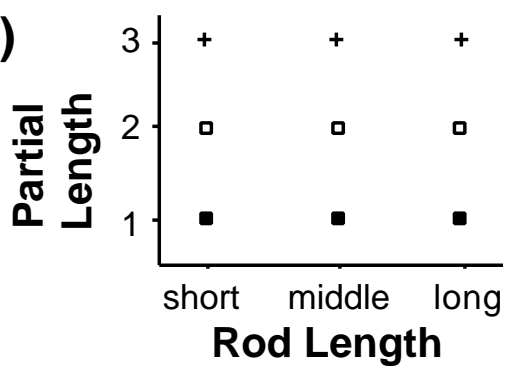

Figure 12. (a) Actual partial length and actual whole length in a typical experiment are positively related. (b) The complete identification experiment requires that the two properties be orthogonal. 
The analysis is a three-step analytic procedure that requires proof of perceptual separability and decisional separability - perceptual effects associated with $\underline{A}$ cannot depend on the level of $\underline{B}$ and the decision about $\underline{\mathrm{A}}$ cannot depend on the perceived value of $\underline{B}$-before perceptual independence can be evaluated. Step 1 evaluates the multiple contingent uncertainty (Garner \& Morton, 1969), the total effects of the stimulus variables $\underline{A}$ and $\underline{B}$ on the response variables $\underline{a}$ and $\underline{b}$. It is analogous to the partial correlation. It essentially looks for $\underline{A}$ to constrain $\underline{a}$ and $\underline{B}$ to constrain $\underline{b} ; \underline{A}$ not to influence $\underline{b}$ beyond the constraint of $\underline{B}$ and $\underline{B}$ not to influence $\underline{a}$ beyond the constraint of $\underline{A}$; and $\underline{a}$ and $\underline{b}$ to be no more related than are $\underline{A}$ and $\underline{B}$ (which, by design, are orthogonal). Importantly, calculations are conducted separately at each level of $\underline{A}_{j} \underline{B}_{j}$ and thus examine the changing relation across all levels of the stimulus. Step 2 tests for marginal response invariance, that is, whether the probability of correctly reporting the level of one variable depends on the level of the other. A response of, for example, $\underline{a} 1$ should be as likely for stimulus $\underline{\mathrm{A}} 1 \underline{\mathrm{B}} 1$ as for stimulus $\underline{\mathrm{A}} 1 \underline{\mathrm{B}} 2$ as for stimulus $\underline{\mathrm{A}} 1 \underline{\mathrm{B}} 3$, and so on. It ensures that decisional criteria do not change across changing conditions of the incongruent stimulus variable. If Steps 1 and 2 show perceptual separability and decisional separability, then Step 3 assesses sampling independence. If the probability of jointly identifying $\underline{A}$ and $\underline{B}$ is equal to the product of their independent identification probabilities, then perceptual independence can be concluded.

These procedures were introduced to the domain of dynamic touch in a $3 \times 3$ design which showed the independence of perceived length and perceived weight (Amazeen, in press). They have since been applied to partial length versus whole length, whole length versus grip position, and grip position versus partial length; all were found to be independent (Cooper, Carello, \& Turvey, in press). In order to examine the consistency between this analysis and our standard tensor functionbased analysis, each of the categorical responses was converted to 1,2, or 3 as appropriate. A numerical mean was thus obtained for each of the nine objects for perception of each of the three properties. Multiple regressions of these means on the tensor components verified the dependencies we have seen with continuous responses: Perceived whole length was constrained by $\underline{I}_{1}$, perceived grip position was constrained by the angle of rotation of the eigenvector, and perceived partial length was constrained by $\underline{I}_{1}$ and the eigenvector rotation (Cooper et al., in press). The joint observations that perceived properties depend not on each other but on different properties of the inertia tensor are a vivid illustration of the perception-information hypothesis at the center of Gibson's ecological approach.

\section{Perceiving the limbs}

As the case of Ian Waterman illustrates, the haptic perceptual system is integral to our ability to do the most fundamental behaviors. In the preceding sections, we have described some of that system's sensitivities with respect to knowing about objects held in the hand, in particular, within a framework for uncovering how rotational dynamics might support those sensitivities. In this section, we argue that the same framework is appropriate for understanding our sensitivities to the dispositions of our limbs. The context for understanding the appropriate rotational dynamics is already in place. The notion of an object being rotated about the wrist joint ${ }^{7}$ is actually a generalization of the notion of the limb itself rotating about the joint (see Figure 1). A set of axes centered at each joint allows a characterization of the body as a tensor field (Figure 13). If the same principles apply to perceiving the limbs as have been shown to apply to perceiving objects, then manipulating the mass distribution of those limbs ought to influence perception of their directions and magnitudes.

One way to test this conjecture is to attach a kind of splint to the arm and weight it symmetrically or asymmetrically with the goal of diverting the arm's eigenvector from coincidence with the longitudinal axis of the arm (Figure 14a). An individual so splinted can be asked to point at a visible target with the whole arm, which is itself occluded from view. As would be expected from a tensorial basis to perceiving the disposition of the limbs (Pagano \& Turvey, 1995), the orientation of the arm during pointing is diverted in the direction consistent with the arm's new eigenvector 


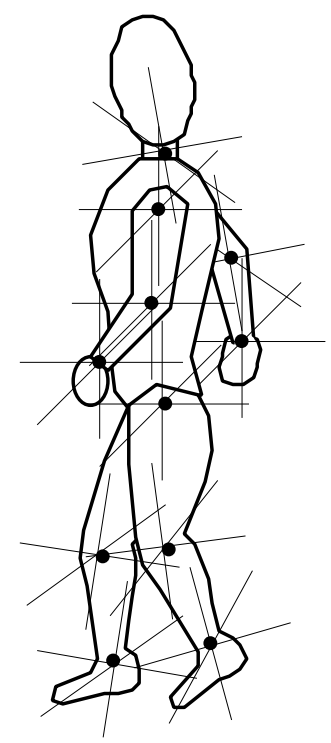

Figure 13. A coordinate system at each joint can be used to define a tensor at that point of rotation. The entire body can be considered a field of tensors.

orientation (Figure 14b). When asked to position a single forearm either parallel to the ground or at a $45^{\circ}$ angle, it is the angle of the eigenvector that satisfies the instruction (Garrett, Pagano, Austen, $\&$ Turvey, 1998). In addition to influencing the perception of the orientation of the arm relative to the environment, a similar manipulation has been used to show that eigenvector direction underlies the perception of the orientation of the two arms relative to each other. When asked to match the orientation of the unseen forearms (Figure 14c), participants instead matched the orientation of the arms' eigenvectors (Pagano, Garrett, \& Turvey, 1996).

To this point, the tensorial perspective on proprioception has focused on limb directions. In principle, however, it should apply to limb magnitudes as well. Finding an experimental task that isn't loaded with demand characteristics is tricky, however. Manipulating the eigenvalues is relatively straightforward: Masses can be attached to the arm (using Velcro exercise weights) either at the wrist or at the elbow. The more distal placement produces a larger $\underline{I}_{1}$ than the more proximal

(a)

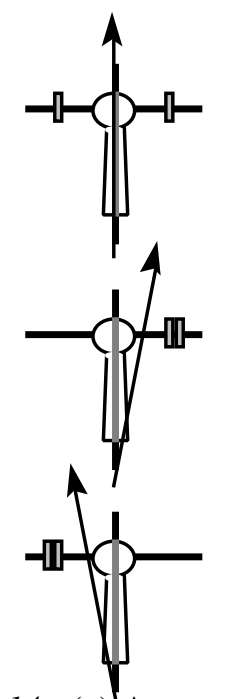

(b)

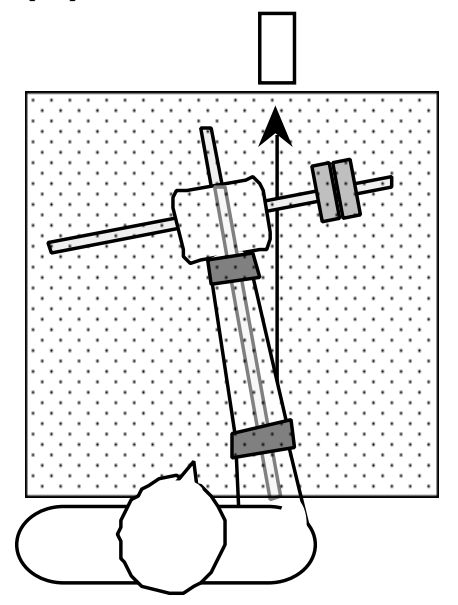

(c)

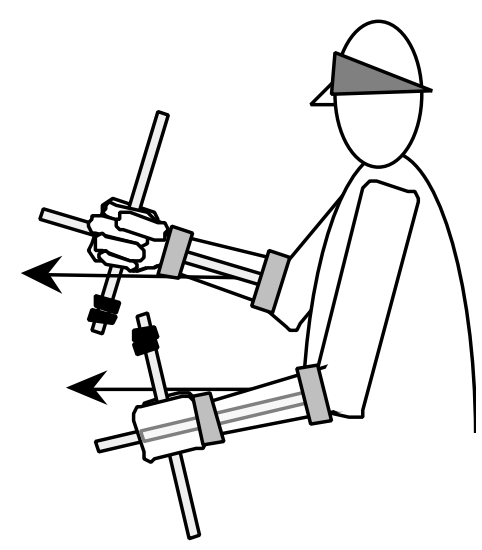

Figure 14. (a) A cross-shaped splint strapped to a participant's arm can be weighted to keep the depicted eigenvector aligned with the arm or to divert it to the right or left. (b) Under the instruction to point at a target with the tip of the cross, a participant instead aligns the depicted eigenvector of the occluded arm with the target. (c) Aligning the unseen forearms is accomplished through the aligning of their eigenvectors. 


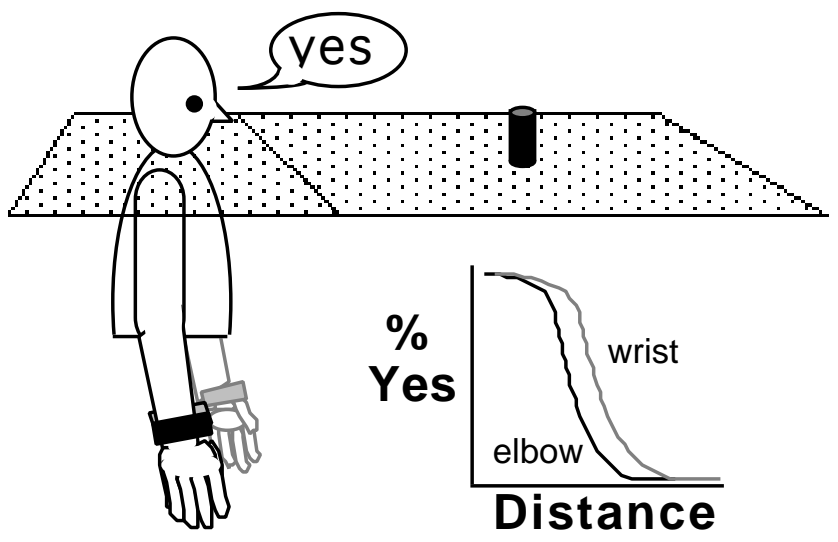

Figure 15. The perceived boundary of reach is farther with weights attached to the wrists rather than the elbows.

placement. The ideal task is one for which arm length is important but that does not require an explicit evaluation of arm length per se. A task examining the affordance for reaching is a promising candidate. In the basic methodology, individuals are asked whether or not a visible target can be reached with the right hand when the arm is extended out from the shoulder, without leaning forward, and without the arms being in view. The perceived boundary of reaching has been shown to distinguish participants with relatively long arms from participants with relatively short arms (for vision, Carello, Grosofsky, Reichel, Solomon, \& Turvey, 1989; for audition, Rosenblum, Wuestefeld, \& Anderson, 1996), suggesting an awareness of arm length. If that awareness is to be considered the "on line" consequence of sensitivity to the arm's inertia tensor, then the reaching boundary should move as a consequence of the eigenvalue manipulation. Preliminary data seem to confirm this basic expectation (Figure 15): Perceived reach is judged to be farther when the participants wear wrist weights than when they wear elbow weights (Anderson \& Turvey, 1998).

The more traditional characterization of proprioceptive abilities is a cognitive account in which the individual has a stored representation of the body. Although the modern incarnation of such a view holds that this representation is more abstract than the "model of oneself" proposed by Head (1920), much detail is assumed to be part of the stored information: spatial and mechanical properties of the limbs, mechanisms and algorithms for how to move and coordinate them (Gurfinkel \& Levick, 1991). Awareness of the location of the hand, for example, is thought to require stored knowledge of limb lengths together with knowledge of joint angle (Craske, Kenny, \& Keith, 1984; Gurfinkel \& Levick, 1979, 1991), either itself sensed or computed from knowing where the limb started and how it was moved. Such computational accounts, however, would seem to demand that the represented body schema be impervious to the kinds of tensorial manipulations to which they are, in fact, susceptible. Once again, that susceptibility does not imply that we do not know where our limbs "really are" but, rather, is rooted in consequences of the body's mass distribution for moving the limbs and maintaining their posture.

\section{Perceiving objects by means of objects}

The kinds of awarenesses on which we have focused-the properties of objects in the hand and the dispositions of the limbs-constitute the subtle but integral contributions that the touch system makes to everyone's daily activities. But touch is also a sense that we think of as substituting when vision is absent. Blind people read by Braille, recognize a face by feel, and detect a curb with a long cane. The last of these is within the realm of dynamic touch that is our focus. Consider the challenge that it provides. We already appreciate that as the cane is held in the hand, its dimensions and directions are specified by the inertia tensor. But then the cane is brought into contact with surfaces and edges and gaps where it scrapes and taps and pokes. Vibrations travel up 
the length of the cane to the hand where the mechanoreceptive neurons are. Tellingly, the surfaces and edges and gaps are felt not in the hand but at the end of the cane (e.g., Vaught, Simpson, \& Ryder, 1968). This separation of perceived properties from the physiology of perception has been referred to as "remote touching" (Katz, 1925/1989) with the implication that the perceiver "projects his sensations of pressure to the tip" of the implement (Békésy, 1967, p. 225). A contrasting characterization was offered by Gibson (1966/1983) who argued that these instances are more properly considered extended haptic perception - the implement simply extends the range over which the perceiver can obtain information. And it is information, not an incidental collection of sensations, that is relevant for perception (Burton, 1992; Turvey \& Carello, 1995a).

Three aspects of this argument should be highlighted. First, the information-perception hypothesis begins with the claim that information about a property is not the property itself. We already appreciate this claim from the research on perceiving object properties by dynamic touch: Information about object length is not object length but the inertia tensor. Second, a corollary assertion is that information about a property is not filtered through the anatomy that happens to pick up that information. Research on perceiving by dynamic touch again provides perspective: An object property such as diameter is influential through its consequences for the mass distribution, not the size of the grasp. Finally, echoing an earlier theme, although skilled cane use is not common in the general population, it is possible because it exploits the very awarenesses that we have described. That people can use a cane to perceive their surrounds should not be surprising; it is logically continuous with perception by other non-neural extensions such as whiskers and claws (Burton, 1993; Gibson, 1966/1983).

What sets perception by non-neural appendages apart is not cognitive but physical-the distance of the probed object from the axis of rotation in the wrist is greater and, if the surface is struck, contact dynamics will be relevant along with rotational dynamics. Let's consider the consequence of axis distance first. Our initial intuitions from a simple tensor characterization might suggest that the farther the axis is from the object being probed, the bigger the object should seem. But we have to be careful because the probing configuration may introduce a second axis of rotation for the target separate from that for the probe, suggesting a field-like structure in which probe inertia modulates target inertia. This latter interpretation is supported by probing experiments in which one rod is used to perceive the length of another. The target rod is supported at one end with an axle and the probe rod is used to "wield" the target or move it up and down about the axle (Figure 16). Target rod length can be perceived under such circumstances and, for a fixed probe inertia, perceived length increases the farther the probe-target contact is from the target's CM (Peck et al., 1996; see Carello, Fitzpatrick, Domaniewicz, Chan, \& Turvey, 1992, for a similar result when the axle-mounted target is "probed" with one finger). This is to be expected if target inertia is defined by resistance to rotation about the axle. Perceived target length is similarly increased by
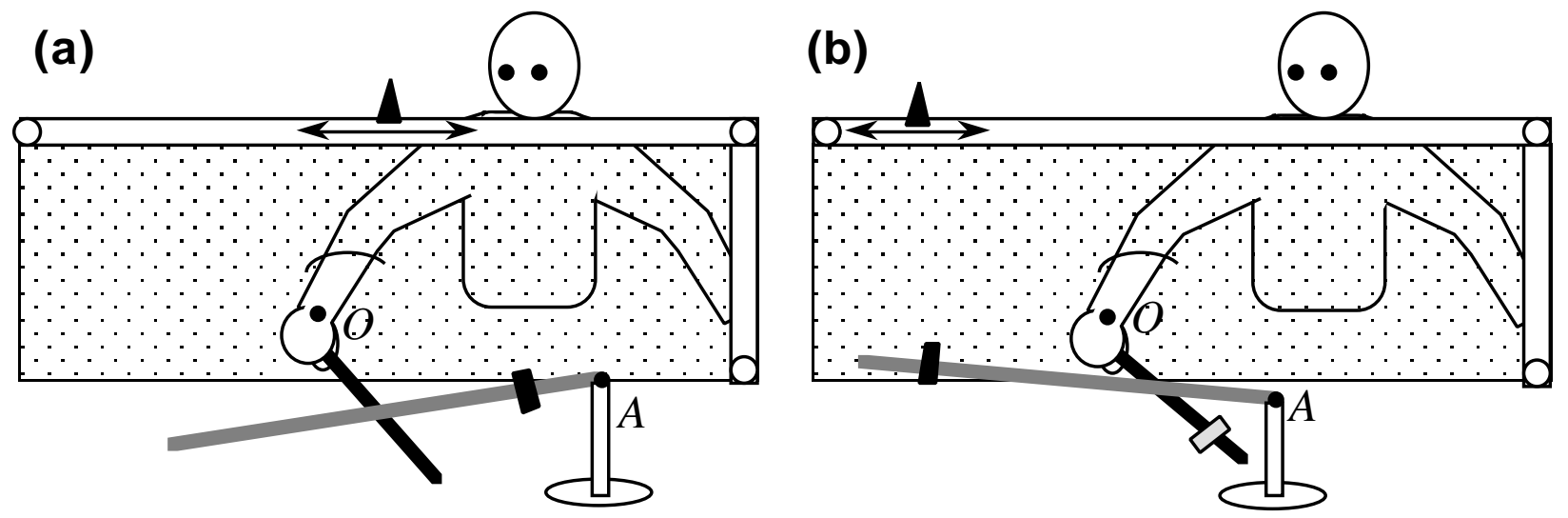

Figure 16. A probe rod is used to wield a target rod (both occluded) that is anchored at one end; a visible marker is positioned to coincide with the tip of the target rod. (a) No mass on the probe, contact at the center of the target, and a mass attached close to the axis $\underline{A}$ at the axle produce a smaller perceived target length than (b) a mass at $3 / 4$ of the probe length from the axis $\underline{\mathrm{O}}$ in the wrist, contact at $1 / 4$ of the target length, and a mass attached far from $\underline{\mathrm{A}}$. 

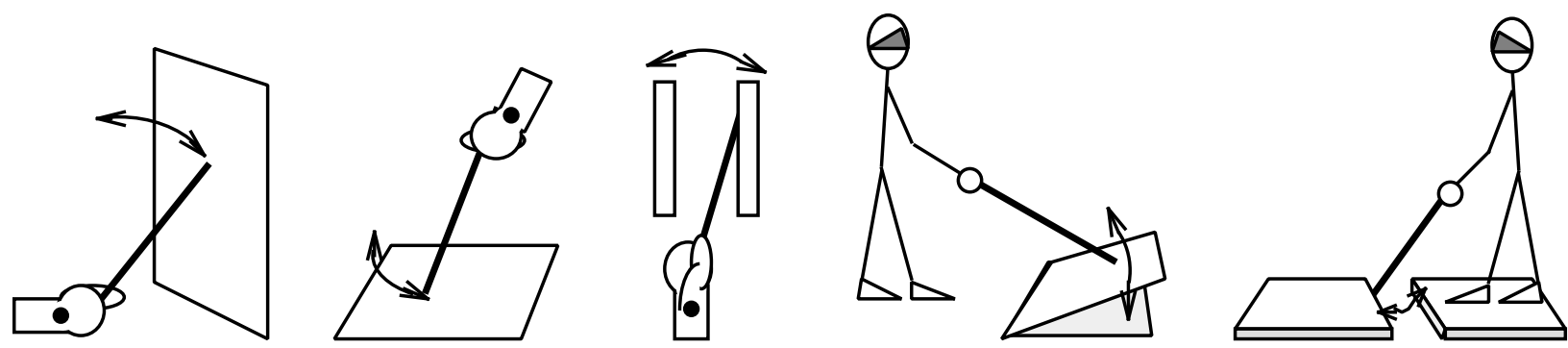

Figure 17. A cane can be used to perceived the distance of a frontal surface by hammering, the distance of a horizontal surface by tapping, the size of a gap by alternating strikes, the support of a slanted surface by scraping, and the crossability of a path gap by alternating taps.

attaching a mass to the target at increasing distances from the axle, again consistent with the axle defined as the center of rotation. Perceived target length is concomitantly increased, however, by attaching a mass to the probe at increasing distances from the wrist. This is, of course, consistent with our initial tensor intuitions. Taken together, these data implicate a higher-order inertial term that incorporates parameters of the probe, the target, and their contact.

Perceiving a movable target object by means of an implement may seem to be a contrived experimental task but the ability is, in fact, implicit in activities such as eating with chop sticks, flipping a burger, and reeling in a fish. Nonetheless, exploring a stationary surface with a probe is more typical of our appreciation of probing as exemplified by cane use. A frontal surface is tapped to gauge stopping distance, a horizontal surface to determine step height; two edges are struck alternately to assess whether the gap between them, if vertical, might be passed through or, if horizontal, might be stepped over (Figure 17). In each of these examples, manipulations of probe characteristics affect not only probe inertia but the reactive forces produced during contact with the surfaces to be perceived. As it happens, this matters to the perceived properties of the surface layout. We first summarize the relevant experiments before considering how such influences might be understood within the ecological framework that guides those experiments.

\section{Perceiving surface layout by means of objects}

It is well established that perceived length of a wielded object is constrained by the rotational inertia of that object. But when the object becomes a probe and contacts a surface repeatedly, as in tapping or striking, impulse forces generate tissue deformations of short duration. These are superimposed on the tissue deformations of relatively longer duration that are brought about by wielding. Just as the varying wielding torques are tied to the varying motions of the object by the invariant inertia tensor, the varying impulse forces are anchored in the rotational dynamics. They are proportional to $\underline{\mathrm{P}}$, the distance of contact from the object's center of percussion, $\underline{\mathrm{CP}}$, which is itself given by the ratio of moment of inertia to static moment. $\underline{P}$ determines how much momentum is transferred to the rotation axis (when $\underline{\mathrm{P}}=0$, no momentum is transferred and contact is said to be at the object's "sweet spot"). Under conditions of contact-be it vertical tapping (Chan \& Turvey, 1991) or horizontal scraping or hammering (Carello, Fitzpatrick, \& Turvey, 1992)-perceived length of a rod is constrained by both $\underline{\mathrm{I}}_{\mathrm{ij}}$, in the form of $\underline{\mathrm{I}}_{\mathrm{xx}}$, and $\underline{\mathrm{P}}$. More poignantly, for rods of different lengths but the same $\underline{I}_{x x}$, perceived length by wielding is the same but perceived length by striking differs, the latter reflecting the parameters of contact (Carello et al., 1992).

When the probe is used to perceive the distance of the surface probed, an additional contact parameter enters prominence. The angle of strike (defined at the wrist) is relevant by virtue of its relationship to the torsion in tissue engendered by the rotation of the forearm during striking. The twisting is arrested when the probe hits the surface and, therefore, torsion is invariant for a given surface location. Under identical conditions of striking, perceived probe length is constrained by $\underline{\mathrm{I} x \underline{x}}$ and $\underline{\mathrm{P}}$ whereas perceived surface distance is influenced by $\underline{\mathrm{I}} \underline{\mathrm{x}}$ (whose contribution is greatly 
(a)

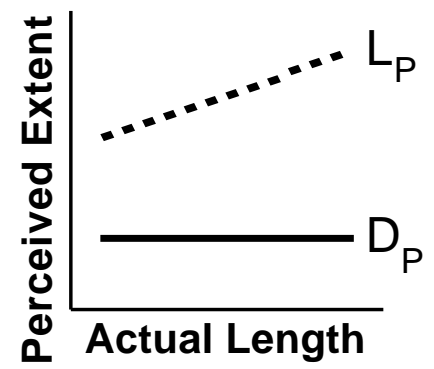

(b)

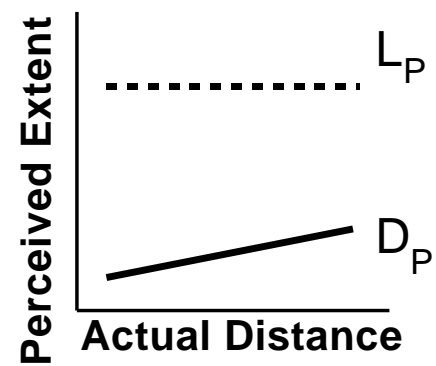

Figure 18. Perceived rod length $\underline{\underline{L}} \underline{P}$ and perceived surface distance $\underline{D} \underline{P}$ are not confused whether variation is in (a) rod length or (b) surface distance.

diminished) and angle of strike. Physically disentangling angle of strike from_P proves to be problematic, however, so that their precise contributions to perceived surface distance are still unclear (cf. Carello et al., 1992, and Chan \& Turvey, 1991). Nonetheless, it is quite clear that perceived probe length is not confused with perceived surface distance (Figure 18): They are distinct in conventional statistical tests, distinct in their functional dependencies, and distinct in the partial correlation analysis advocated earlier for other selective perception experiments. Recently, moreover, the complete identification experiment has shown perceived surface distance, perceived probe length, and perceived angle of strike to be independent (Cooper, Carello, \& Turvey, 1998).

The goal of these kinds of investigations, ultimately, is to identify the physical invariant that is extracted during the act of exploring a surface layout. A programmatic examination of perceived aperture size exemplifies this endeavor (Barac-Cikoja \& Turvey, 1991, 1993, 1995). In the typical experimental paradigm, a gap is explored by striking its inner edges with the hand-held rod. The rod's inertia 8 is manipulated as are the size of the gap and its distance of contact from the axis of rotation, with designed consequences for the angle of excursion of the rod (e.g., angle can be held constant with different combinations of gap size and distance). In addition to defining the relevant physics, the geometric arrangement allows an examination of the trigonometric relation at the heart of the classic law of size constancy in vision-perceived size equals perceived distance times perceived angle. (In this regard, it should also be noted that variation in probe inertia offers a manipulation of the medium in a way that has not been possible in the visual perception of size.) Apart from the unsurprising fact that perceived aperture size increases with actual aperture size, the basic observations from dynamic touch are not consistent with the law of size constancy: Perceived aperture size decreases with contact distance and with $\underline{I}_{y y}$, size discrimination decreases at a rate dictated by contact distance, and perceived aperture distance is statistically distinct from perceived aperture size. Rather than being understood as an instance of a coupling between two percepts, departures from size constancy were thoroughly predicted by a collective dimensionless quantity, dubbed $\underline{\lambda}$, that for a given rod, aperture, and distance, is invariant over muscular forces, resultant impulsive torques, and motions (Figure 19). And, consistent with the characterization of extended haptic perception as indifferent to particular sensations, $\underline{\lambda}$ is the relevant quantity whether the probe is wielded from the wrist, elbow, or shoulder or, for that matter, whether the probe is the index finger itself.

We began this section by noting the behavioral possibilities that probing can uncover for caneusers. Some examples of such affordances have been examined in an experimental setting. For example, participants can explore a $1 \mathrm{~m}^{2}$ slanted surface with a single probe from a distance of $1 \mathrm{~m}$ in order to determine whether it would support stable upright stance (Fitzpatrick, Carello, Schmidt, \& Corey, 1994). This affordance is judged accurately-indeed, a comparison of haptic exploration with visual exploration revealed that the transition between a surface perceived as supporting stance and not supporting stance is the same. Moreover, both showed an increase in exploration time near the transition, with the only difference being that vision was faster. Although the probe was not manipulated in this investigation, it was in a separate affordance evaluation, that concerned whether a gap in the ground surface is crossable (Burton, 1992). An adjustable ground surface can be 
(a)

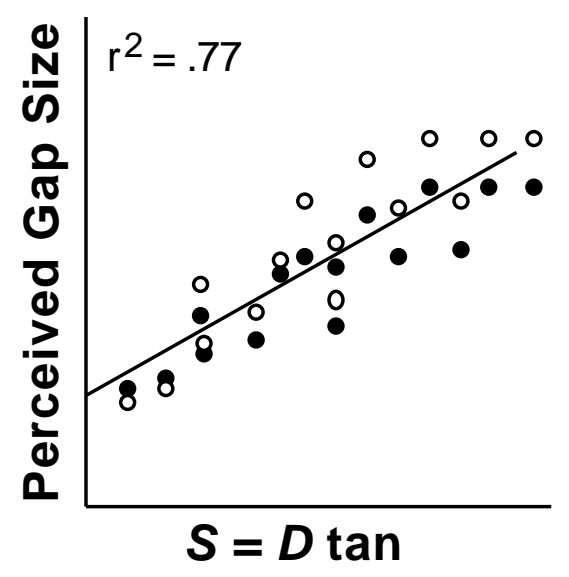

(b)

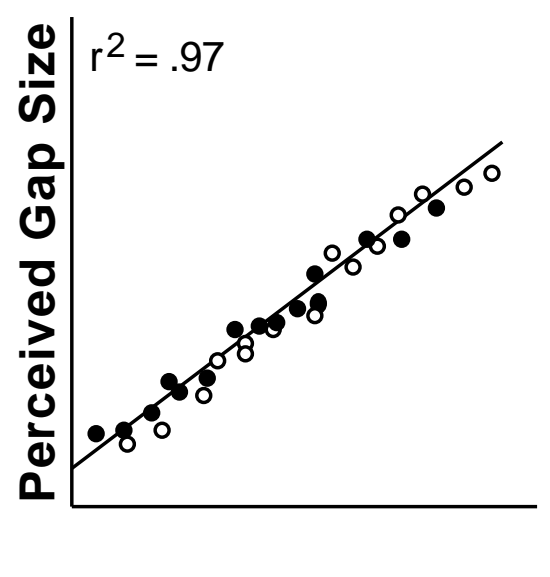

Figure 19. The prediction of perceived gap size by striking as a function of (a) the geometric law governing image formation that underlies the supposed coupling of size and distance in vision, and (b) $\lambda$, an invariant of the time-varying dynamics of probing.

constructed as a walkway of platform segments that, when abutted, produce a completely uninterrupted substrate or, when systematically separated, produce a gap of varied sizes anywhere along the walkway. A blindfolded participant can explore a gap with a probe while standing still on one side of it or after encountering it while walking along the walkway. In either case, the assessment is whether or not the gap could be stepped over. The largest crossable gap is wider for taller individuals than for shorter ones and that difference is reflected in their judgments. Once scaled by their respective leg lengths, however, the perceived boundary between crossable and not crossable is the same. Interestingly, and in contrast with preceding studies of probing, subsequent manipulations of probe $\underline{I}_{\mathrm{ij}}$ were relatively inconsequential apart from the influence that probe length had on how much an individual had to lean to reach the other side of the gap with the probe.

The notion that probe characteristics, whether inertial or geometric, contribute to perception of surface properties deserves comment. Although a probe allows reliable perception of a variety of properties, it is not strictly transparent. Rather than considering this intrusive on perception, however, we have simply noted where the mechanics of contact allow such a contribution. In this regard, it is important to note that a probe is not simply a medium for conducting vibrations; it is a functional part of the probe-target system that allows perception to guide effective action (Peck et al., 1996). It may be appropriate to speak of functional transparency (cf. Shaw \& Bransford, 1977) to the extent that we better understand exactly what is being perceived. We have already lobbied against hypostatizing simple mathematical quantities such as length and width and promoted affordances as an alternative. We now pursue this notion explicitly as it relates to dynamic touch.

\section{Dynamical symmetry and affordance: The perception of heaviness}

In laying out a framework for studying dynamic touch, we noted earlier that the characterization of objects in terms of rotational dynamics is quite germane to how those objects can be used. The uncommon English word "wieldy" serves as the positive pole for which the more common "unwieldy" anchors the difficult-to-use end. This intuitive dimension can be given a formal understanding in terms of the relative magnitudes of the eigenvalues and, relatedly, how an object so described would respond to torques applied to rotate it about the axes centered in the wrist. Three dynamically distinct classes of objects can be identified based on the symmetry of their inertia ellipsoids relative to $\underline{\mathrm{O}}$ (Hestenes, 1986): All three moments are equal for a centrosymmetric object (the 
ellipsoid is spherical); two moments are equal and one is distinct from them for an axially symmet-

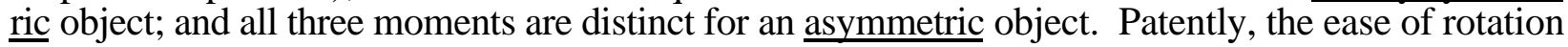
will be less axis-dependent for centrosymmetric objects than the other two and this has consequences for how these objects can be used. For an object that is centrosymmetric about $\underline{\mathrm{O}}$, the dynamics about any given axis of rotation through $\mathrm{O}$ is equal to the dynamics about any other axis of rotation through $\underline{O}$. Simply put, the object is as easy (or as hard) to turn in any one direction as it is in any other. In contrast, the dynamics of an object that is asymmetric relative to $\underline{\mathrm{O}}$ depend dramatically on the chosen axis. It is much harder to turn about the principal axis corresponding to the maximal moment $\underline{I}_{1}$, and much easier to turn about the principal axis corresponding to the minimal moment $\underline{I}_{3}$, than it is about any other axis. From the perspective of muscular synergies and coordination, controlling the motions of an asymmetric object is more challenging than controlling the motions of a centrosymmetric object. Imagining good and bad shapes (and orientations of them) for rolling, spinning, hammering, swatting, scooping, and so on, gives insight into how dynamical symmetry might be used to identify different affordances.

The significance of dynamical symmetry is highlighted by Amazeen and Turvey's (1996) observation that the perceived heaviness of a freely wielded occluded object of a fixed mass and fixed linear dimensions (a "tensor object," see Figure 20a) decreases as its eigenvalues become more nearly identical (Figure 20b), that is, as its dynamics approximate more closely those of a centrosymmetric object. The eigenvalues of Amazeen and Turvey's objects were manipulated to conform to the (previously unmeasured) changes in the pattern of eigenvalues that occur across a typical set of same-weight objects used to demonstrate the size-weight illusion (e.g., Stevens \& Rubin, 1970). The lesson of Amazeen and Turvey's finding is that the haptic size-weight illusion is, in fact, an example of specificity; namely, when weight and other factors are constant for a given set of objects, perceived heaviness is a function of their dynamical symmetry.

It is apparent that, in the most general case, the affordance to which haptically perceived heaviness refers must be inclusive of an object's inertia tensor and its mass-its inertia for rotation and translation (Amazeen, 1997) — both taken in reference to the properties of the human movement system. A linear combination of the two types of inertia in determining perceived heaviness would be most consistent with the law of rigid body motion. Forces (proportional to mass) and torques (proportional to the inertia tensor) combine linearly in such a way that the rate of change of the combination determines the summed linear and angular momentum of a rigid object (Hestenes, 1986, p. 426). Consistent with the preceding, experiments reveal that perceived heaviness is proportional to mass together with two parsings of the inertia tensor, one (a measure of inertia ellipsoid volume, $\underline{V}=4 \pi / 3\left[\underline{I}_{1} \times \underline{I}_{2} \times \underline{I}_{3}\right]^{-1 / 2}$ ) relevant to the mean level of torque needed to wield an object and the other (a measure of dynamic symmetry, $\underline{S}=2 \underline{I}_{3} /\left(\underline{I}_{1}+\underline{I}_{2}\right)$ relevant to how those torques should be directed (Shockley, Carello, \& Turvey, under review). The deep significance of $\underline{S}$
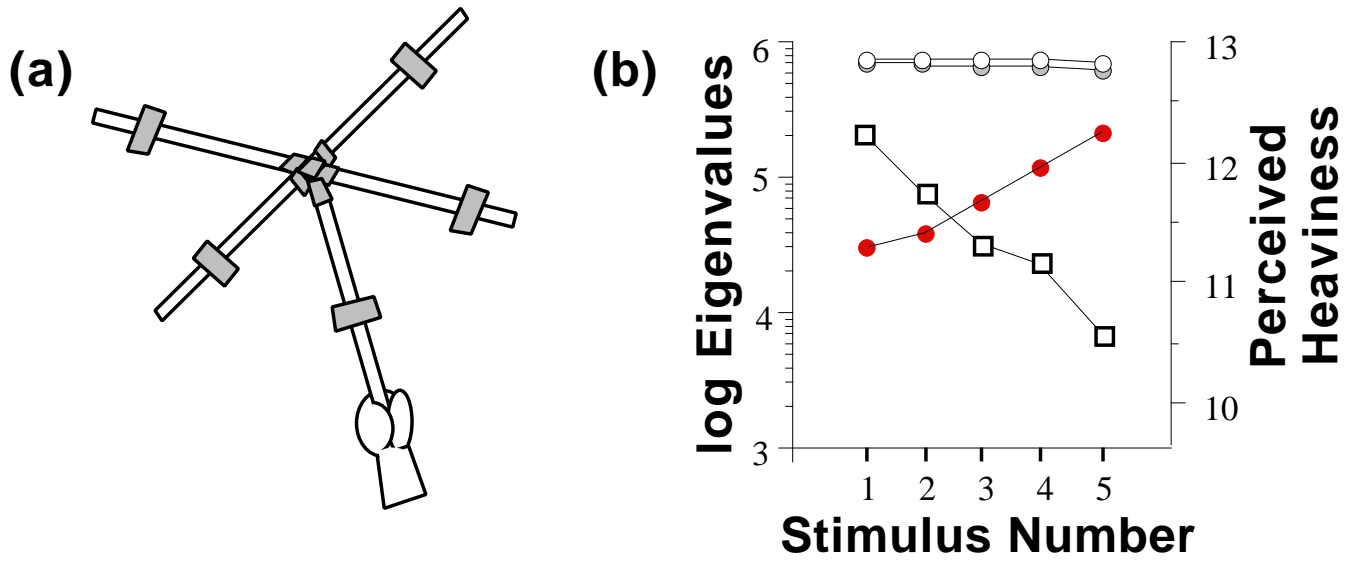

Figure 20. (a) A "tensor object" with attached masses whose positions can be adjusted independently so as to create specific tensors. (b) As the eigenvalues of the tensor (circles) become more nearly identical, perceived heaviness (squares) decreases. 
and $\underline{V}$ is that they are physical characterizations of an object's resistance to rotational acceleration (its inertia tensor) taken in reference to the human movement system. As such, they offer the kind of biologically plausible understanding of perception by dynamic touch that promises to bring welldefined functional contexts to the core of an extensive body of data.

The relevance of this lesson for understanding haptic perception, and for perceptual theory more generally, lies in the fact that dynamical symmetry is an objective, real, physical property defined in the complementation of properties of the object and properties of the neuromuscular, biomechanical system that moves the object. It is a valuable example of the special kind of physical property identified in Gibson's theory of affordances. Indeed, when judgments of heaviness are put in a functional context - how heavy should a given sized object be for optimum throwing - the characteristic size-weight "illusory pattern" is produced (Bingham, Schmidt \& Rosenblum, 1989). In the functional context, however, the connection between size and weight is motivated, quite unlike their arbitrary coupling in more inferential accounts. In particular, size and weight have complementary consequences for timing the spring-like action at the wrist during hefting. An invariant phase between wrist and elbow accompanies the hefting of preferred throwing objects (Bingham et al. 1989). The implication is that relating an affordance-based physical characterization of an object to the movement system that controls the afforded behavior may well provide the most principled characterization of one of the oldest phenomena in experimental psychology (Weber, 1834/1978).

\section{Parallels from other perceptual systems}

Throughout this chapter, we have emphasized that perception is constrained by information rather than being dependent on sensations. This is, perhaps, most apparent in those examples of the equivalence of extended haptic perception across differing conditions of tissue contact: Lifting a rod or probing a gap with a finger is equivalent to lifting that same rod or probing that same gap with a rod. But the point is a general one, not limited to the success of probing. The physical constraints on perceived length are the same whether the object is wielded about the wrist, the elbow, the shoulder or all three joints at once (Pagano, Fitzpatrick, \& Turvey, 1993). The physical constraints on perceived partial length (Turvey et al., 1998) or on perceived grip position (Pagano et al., 1994) are the same whether the object is grasped in the hand or pinched by the thumb and fingers. Even when the rod is held as still as possible, the physical constraints on perceived length are the same whether it is supported by the tissue of one hand, two hands, or a hand and a knee (Carello et al., 1992). ${ }^{9}$ In all of the preceding cases, the sensations in the tissues of the hand and arm vary widely across the situations as well as during the act of exploration. What does not change-and what, therefore, allows perception-is information.

One implication of the perception-information hypothesis, therefore, is that the varieties of tissue contacts ought to reveal the same physical constraints on perception. In highlighting the irrelevance of sensations, the hypothesis can be generalized still further: Information is not tied to any particular receptor surface nor to any particular energy medium. Information is about environmental properties of relevance to behavior; it is not about the piece of anatomy that happens to register that information. To the extent that rotational inertia can structure other energy media, therefore, perception of properties such as length, width, and shape ought to be constrained by rotational inertia in the same way that we have shown for dynamic touch. Sound waves are structured, for example, by vibrations set up by interacting materials, interactions such as the impact of an object and a surface. The amplitude, frequency, and damping of those vibrations are influenced by the inertia, stiffness, and elasticity of the materials (Gaver, 1993).

Experiments modeled after those from dynamic touch verify that perceived rod length on the basis of sound is constrained by the inertia tensor (Figure 21a; Carello, Anderson, \& Kunkler-Peck, 1998). The rods - whose number, length range, material, and radii are unknown to listeners beforehand - are allowed to drop and bounce on a surface several times. Magnitude production is used to indicate the extent of each heard rod. For a homogeneous set of wooden rods $(30-120 \mathrm{~cm}$ 

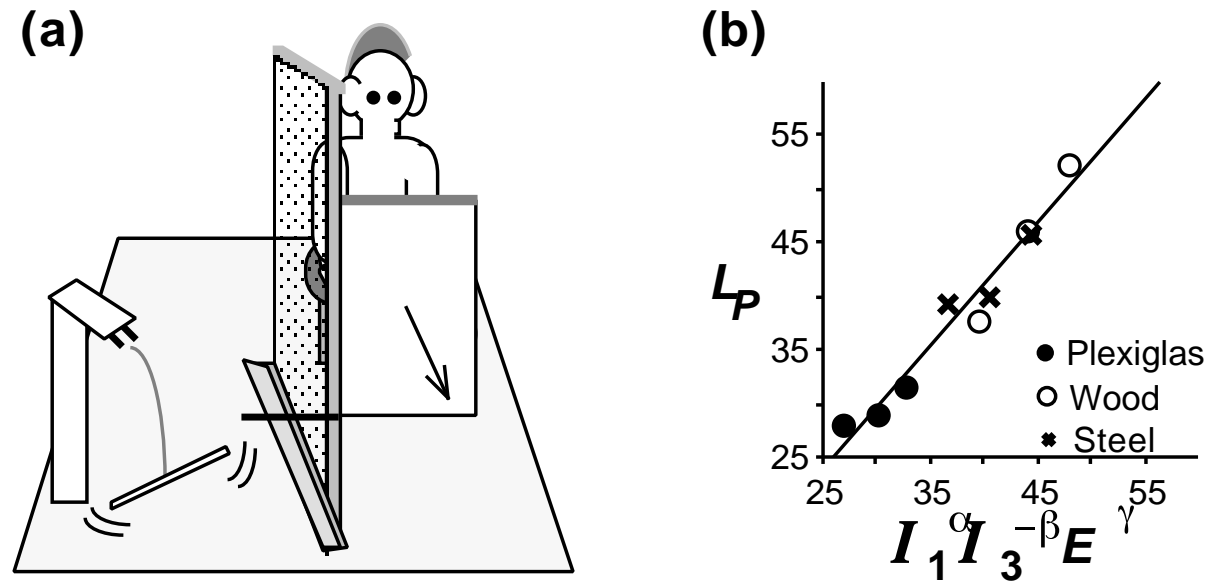

Figure 21. (a) A lever is turned to let a rod, supported at its $\underline{\mathrm{CM}}$, drop onto a hard surface as a visible surface is positioned to be as far away as could be reached with the rod if it extended directly in front of the listener. (b) Perceived length $\underline{L}_{P}$ was in the scale of actual length $\underline{L}_{A}$. (c) When the dropped rods included various material densities, the tensor account is augmented with an elasticity component $\underline{E}$.

long and $.64 \mathrm{~cm}$ radius), perceived length is in a definite scale $(24-95 \mathrm{~cm})$, constrained by $\underline{I}_{1}$ raised to the $1 / 3$ power. Rods that are shorter and thinner than the preceding $(10-40 \mathrm{~cm}$ long and $.16 \mathrm{~cm}$ radius) are also perceived in a definite scale appropriate to their range $(14-27 \mathrm{~cm})$. The two sets together are captured by the maximum and minimum eigenvalues, raised to a positive and negative power, respectively (Carello et al., 1998). This is reasonably remarkable. Consider that we have two groups of listeners who know nothing about their own rods beforehand, who do not know that there are two groups of listeners or that there are two sets of rods whose lengths overlap only slightly. Yet the two groups of listeners confine themselves to using that part of the report apparatus that is appropriate to the rods they hear. And the physical constraint on that perception is the same physical constraint that has been shown to constrain perceived length by dynamic touch. Interestingly, however, this physical constraint is not adequate to completely characterize perceived length when the material of the rods is varied (Anderson, Peck, \& Carello, 1996); an elasticity component must be included (Figure 21b). In contrast to the experiments in dynamic touch after which these were modeled (Fitzpatrick et al., 1994), it seems that material density is relevant to more than the mass distribution of the object. Material density is also relevant to the restoring forces by which the object recovers from impact. Elasticity as such has not been examined in dynamic touch, where we have avoided objects that bounce or spring when wielded or struck. The close parallel between perceiving an object by the sounds produced during its impact with a surface and perceiving an object by the tissue deformations produced during wielding suggests that such an examination might prove fruitful.

The rod dropping experiments address perception of an object during impact with a surface. The object is manipulated while the surface is held constant. But the surface is also one of the interacting materials in the impact event. Its properties contribute to the structured sound waves and, therefore, ought to be available to perception as well. Important among the properties of a surface is its two-dimensional structure. It has width as well as length. It has shape. The rod dropping paradigm was limited to long, thin cylinders. Rod width (radius) was manipulated because of its consequences for $\underline{I}_{\mathrm{ij}}$ but listeners were asked only about the lengths of those cylinders. Returning to dynamic touch for inspiration, however, we know that shape can be perceived categorically (Burton et al., 1990) and that width as well as length can be perceived metrically, all of these constrained by the inertia tensor (Turvey et al., 1998). For parallel questions in ecological acoustics, object-surface impact is again the sound-producing event of choice. This time the object, a pendulum bob (a metal sphere dangling from fishing line) is held constant and the surfaces it strikes (thin plates of different shapes and materials) are varied (Figure 22a). For the shape 


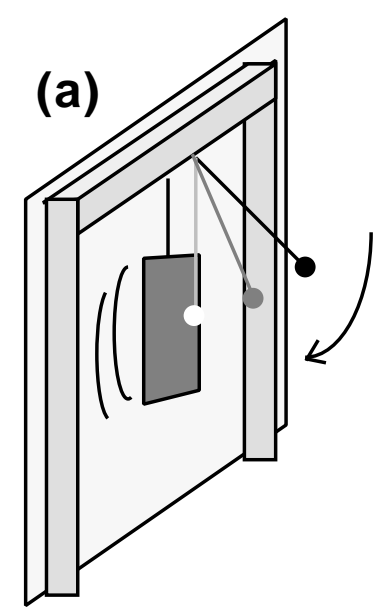

(b)

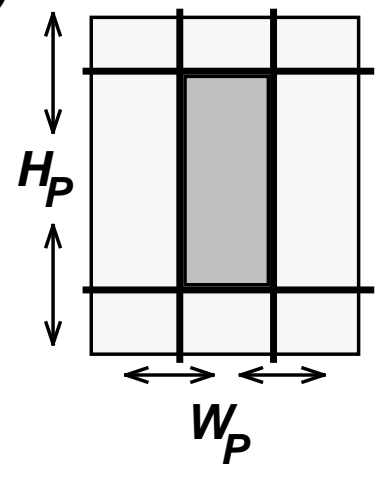

(c)

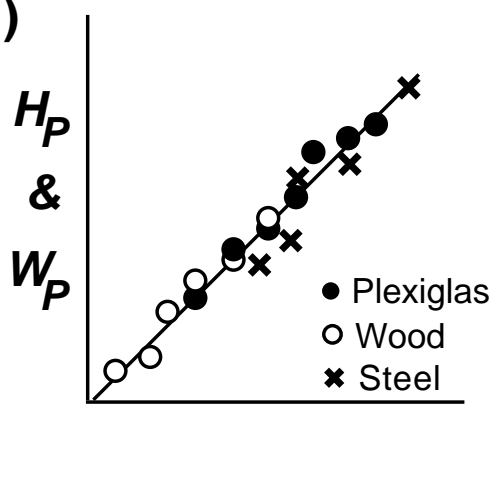

Figure 22. (a) A thin plate suspended by fishing line is struck by a pendulum bob (the plates can vary in material density, size, and shape). (b) For rectangular plates, perceived size is indicated by adjusting the separation between two horizontal dowels to match perceived height $\underline{\mathrm{HP}}$, and between two vertical dowels to match perceived width $\underline{\mathrm{WP}}$. (This report apparatus occludes the plates from the Tisteners' view.) (c) $\mathrm{HP}_{\mathrm{P}}$ and $\mathrm{WP}_{\mathrm{P}}$ are a single-valued function of a complex parameter $\underline{\Gamma}$ that incorporates inertial and elastic quantities.

perception experiments, listeners see cardboard silhouettes of the three possible shapes (circle, triangle, and square) and point at or name what they hear when the surface is struck (three times in succession). These shapes are distinguished categorically, whether the plates are wood, steel, or Plexiglas (Kunkler-Peck, 1997; Kunkler-Peck \& Turvey, in press). The metrical question is asked with respect to rectangles whose surface areas are the same but whose proportions can be characterized as squares, tall rectangles, or wide rectangles (this is, therefore, also a matter of shape perception). Perceived height and width are indicated by adjusting the positions of movable edges that are visible to the listener (Figure 22b). Heights and widths of rectangles of different proportions and materials are perceived metrically, constrained by a physical quantity (Figure 22c) that incorporates inertial and elastic components in the form of the two-dimensional wave equation (Kunkler-Peck \& Turvey, in press). Again, this is remarkable. A variety of plates are struck during the course of the experiment and they sound different from one another. Not only can they be distinguished from one another but they are distinguished reliably, systematically, and at a definite scale that is rationalized by the physics of the impact event.

The preceding should be understood within the same ecological law framework that was introduced earlier. An event lawfully structures the acoustic array; that structure specifies the event that generated it. Even though the listeners are naive to relying solely on sound (as the wielders are naive to relying solely on tissue deformation) reliably structured energy media permit perceivers to detect the relevant information. Because there is nothing arbitrary about the structured sound, there is nothing arbitrary about the mapping of perceived properties from that sound. Although we find it remarkable that listeners are as good as they are in these kinds of experiments, perhaps we should not. 


\section{Epilogue}

"You may use any degrees of freedom you like in describing a physical system, but if you use the wrong ones, you'll be sorry."

(Weinberg, 1983)

A central theme of the work on dynamic touch is discovering the appropriate quantities to manipulate and to index perception. This is a problem of identifying those properties of the environment to which perception really refers. We have already asserted the appropriateness of an affordance description of the environment over a description that reifies elementary physical variables or convenient linguistic labels (cf. Bingham, 1988). The issue is not unrelated to the question of the proper function (Millikan, 1984, 1993) of the haptic perceptual system. A proper function not only entails a system's job description but, more importantly, its raison d'être. With respect to the haptic perceptual system, a reasonable guess is that its proper function is registering resistances to rotational and linear acceleration in a form and a manner that satisfy the requirements for generating the muscle tensile states that move an object in the desired way. The physical basis of any property perceived by dynamic touch, therefore, requires consideration of the haptic perceptual system's role in controlling actions (the system's proper function) and the relation of an object's inertial properties to properties of the human movement system (the object's affordance). At least that is the gambit we have chosen to pursue. In the context of Weinberg's admonition, this seems the most principled gambit available.

\section{Acknowledgments}

Preparation of this manuscript was supported by National Science Foundation Grant SBR 9709678. We thank all of the students at the Center for the Ecological Study of Perception and Action whose work is summarized here. We are especially grateful to H. Yosef Solomon, whose early theoretical insights laid the foundation for the development of this research program.

\section{References}

Amazeen, E. L. (1997). The effects of volume on perceived heaviness by dynamic touch: With and without vision. Ecological Psychology, 9, 245-264.

Amazeen, E. L. (in press). Perceptual independence of size and weight by dynamic touch. Journal of Experimental Psychology: Human Perception and Performance.

Amazeen, E., \& Turvey, M. T. (1996). Weight perception and the haptic size-weight illusion are functions of the inertia tensor. Journal of Experimental Psychology: Human Perception and Performance, 22, 213-232.

Anderson, K. L., \& Turvey, M. T. (May, 1998). Inertial eigenvalues may influence perceived reach. Poster presented at the Spring meeting of the North American Society for Ecological Psychology, Hartford, CT.

Anderson, K., Peck, A. J., \& Carello, C. (March, 1996). Role of the inertia tensor in perceiving length by sound. Poster presented at the Spring meeting of the North American Society for Ecological Psychology, Hartford, CT.

Ashby, F. G., \& Townsend, J. T. (1986). Varieties of perceptual independence. Psychological Review, 93, 154-179.

Barac-Cikoja, D., \& Turvey, M. T. (1991). Perceiving aperture size by striking. Journal of Experimental Psychology: Human Perception and Performance, 17, 330-346. 
Barac-Cikoja, D., \& Turvey, M. T. (1993). Haptically perceiving size-at-a-distance. Journal of Experimental Psychology: General, 122, 347-370.

Barac-Cikoja, D., \& Turvey, M. T. (1995). Does perceived size depend on perceived distance? An argument from extended haptic perception. Perception \& Psychophysics, 57, 216-224.

Békésy, G. von (1967). Sensory inhibition. Princeton, NJ: Princeton University Press.

Bell, C. (1826). On the nervous circle which connects the voluntary muscles with the brain. Philosophical Transactions of the Royal Society, 116, 163-173.

Bingham, G. P. (1988). Task-specific devices and the perceptual bottleneck. Human Movement Science, 7, 225-264.

Bingham, G. P. (1993). Perceiving the size of trees: Forms as information about scale. Journal of Experimental Psychology: Human Perception \& Performance, 19, 1139-1161.

Bingham, G. P., Schmidt, R. C., \& Rosenblum, L. D. (1989). Hefting for a maximum distance throw: A smart perceptual mechanism. Journal of Experimental Psychology: Human Perception and Performance, 15, 507-528.

Burton G. (1992). Nonvisual judgment of the crossability of path gaps. Journal of Experimental Psychology: Human Perception and Performance, 18, 698-713.

Burton, G. (1993). Non-neural extensions of haptic sensitivity. Ecological Psychology, 5, 105124.

Burton, G., \& Turvey, M. T. (1990). Perceiving the lengths of rods that are held but not wielded. Ecological Psychology, 2, 295-324.

Burton, G., \& Turvey, M. T. ( 1991). Attentionally splitting the mass distribution of a hand-held rod. Perception \& Psychophysics, 50, 129-140.

Burton, G., Turvey, M. T., \& Solomon, H. Y. (1990). Can shape be perceived by dynamic touch? Perception \& Psychophysics, 48, 477-487.

Carello, C., Anderson, K. L., \& Kunkler-Peck, A. J. (1998). Perception of object length by sound. Psychological Science, 9, 211-214.

Carello, C., Fitzpatrick, P., Domaniewicz, I., Chan, T-C., \& Turvey, M. T. (1992). Effortful touch with minimal movement. Journal of Experimental Psychology: Human Perception and Performance, 18, 290-302.

Carello, C., Fitzpatrick, P., \& Turvey, M. T. (1992). Haptic probing: Perceiving the length of a probe and the distance of a surface probed. Perception \& Psychophysics, 51, 580-598.

Carello, C., Fitzpatrick, P., Flascher, I., \& Turvey, M. T. (1998). Inertial eigenvalues, rod density, and rod diameter in length perception by dynamic touch. Perception \& Psychophysics, 60, 89100.

Carello, C., Grosofsky, A., Reichel, F. D., Solomon, J., \& Turvey, M.T. (1989). Visually perceiving what is reachable. Ecological Psychology, 1, 27-54.

Carello, C., Santana, M. V., \& Burton, G. (1996). Selective perception by dynamic touch. Perception \& Psychophysics, 58, 1177-1190.

Carello, C., \& Turvey, M. T. (1985). On vagueness and fictions as cornerstones of a theory of perceiving and acting. Cognition and Brain Theory, 7, 247-261.

Chan, T-C. (1994). Haptic perception of partial-rod lengths with the rod held stationary or wielded. Perception \& Psychophysics, 55, 551-561.

Chan, T-C. (1995). The effect of density and diameter on haptic perception of rod length. Perception \& Psychophysics, 57, 778-786.

Chan, T-C., \& Turvey, M. T. (1991). Perceiving the vertical distances of surfaces by means of a hand-held probe. Journal of Experimental Psychology: Human Perception and Performance, $17,347-358$.

Cole, J. (1995). Pride and a daily marathon. Cambridge, MA: MIT Press.

Cooper, C., Carello, C., \& Turvey, M. T. (May, 1998). Probe length, distance, and angle, are perceptually independent. Poster presented at the Spring meeting of the North American Society for Ecological Psychology, Hartford, CT.

Cooper, M., Carello, C., \& Turvey, M. T. (in press). Perceptual independence of whole length, partial length, and hand position in wielding a rod. Journal of Experimental Psychology: Human Perception and Performance. 
Craske, B., Kenny, F. T., \& Keith, D. (1984). Modifying an underlying component of perceived arm length: Adaption of tactile location induced by spatial discordance. Experimental Psychology: Human Perception and Performance, 10, 307-317.

Epstein, W. (1977). What are the prospects for a higher-order stimulus theory of perception? Scandinavian Journal of Psychology, 18, 164-171.

Epstein, W. (1982). Percept-percept coupling. Perception, 11, 75-83.

Fitzpatrick, P., Carello, C., Schmidt, R. C., \& Corey, D. (1994). Haptic and visual perception of an affordance for upright posture. Ecological Psychology, 6, 265-287.

Fitzpatrick, P., Carello, C., \& Turvey, M. T. (1994). Eigenvalues of the inertia tensor and exteroception by the "muscle sense." Neuroscience, 60, 551-568.

Garner, W. R., \& Morton, J. (1969). Perceptual independence: Definitions, models and experimental paradigms. Psychological Bulletin, 72, 233-259.

Garrett, S. R., Pagano, C. C., Austin, G. P., \& Turvey, M. T. (1998). Spatial and physical frames of reference in positioning a limb. Perception \& Psychophysics, $\underline{60}, 1206-1215$.

Gaver, W. W. (1993a). What in the world do we hear? An ecological approach to auditory event perception. Ecological Psychology, 5, 1-29.

Gibson, J. J. (1983). The senses considered as perceptual systems. Prospect Heights, IL: Wavelan. (Original work published in 1966)

Gibson, J. J. (1979). The ecological approach to visual perception. Boston: Houghton Mifflin.

Gogel, W. C. (1977). The metric of visual space. In W. Epstein (Ed.), Stability and constancy in visual perception: Mechanisms and processes (pp. 129-181). New York: Wiley.

Gurfinkel, V. S., \& Levick, Y. S. (1979). Sensory complexes and sensorimotor integration. Human Physiology, 5, 269-281.

Gurfinkel, V. S., \& Levick, Y. S. (1991). Perceptual and automatic aspects of the postural body scheme. In J. Paillard (Ed.) Brain and space (pp. 147-162). New York: Oxford University Press.

Head, H. (1920). Studies in neurology, Vol. 2. London: Oxford University Press.

Hestenes, D. (1986). New foundations for classical mechanics. Dordrecht, The Netherlands: Kluver Academic Publishers.

Hestenes, D. (1994a). Invariant body kinematics: I. Saccadic and compensatory eye movements. Neural Networks, 7, 79-88.

Hestenes, D. (1994b). Invariant body kinematics: II. Reaching and neurogeometry. Neural Networks, 7, 65-77.

Katz, D. (1989). The world of touch (L. Krueger, trans.). Hillsdale, NJ: Erlbaum. (Original work published 1925).

Kugler, P. N., \& Turvey, M. T. (1987). Information, natural law and the self-assembly of rhythmic movement. Hillsdale, NJ: Erlbaum.

Kunkler-Peck, A. J. (1997). Can you hear the shape of a drum? Doctoral dissertation, University of Connecticut, Storrs, CT.

Kunkler-Peck, A. J., \& Turvey, M. T. (in press). Hearing shape. Journal of Experimental Psychology: Human Perception and Performance.

Lederman, S. J., Ganeshan, S. R., \& Ellis, R. E. (1996). Effortful touch with minimum movement: Revisited. Journal of Experimental Psychology: Human Perception and Performance, 22, 851868.

Maddox, W. T., \& Ashby, F. G. (1996). Perceptual separability, decisional separability, and the identification-speeded classification relationship. Journal of Experimental Psychology: Human Perception and Performance, 22, 795-817.

Michaels, C. F., \& Carello, C. (1981). Direct perception. Englewood Cliffs, NJ: Prentice Hall.

Michaels, C. F., Carello, C., \& Shapiro, B. (1985). Issues in event perception: Report of the perception work group. In W. H. Warren \& R. E. Shaw (Eds.), Persistence and Change: Proceedings of the First International Conference on Event Perception. Hillsdale, NJ: Erlbaum.

Millikan, R. (1984). Language, thought and other biological categories. Cambridge, MA: MIT Press. 
Millikan, R. (1993). White queen psychology and other essays for Alice. Cambridge, MA: MIT Press.

Pagano, C. C., Carello, C., \& Turvey, M. T. (1996). Extero- and exproprio-perception by dynamic touch are different functions of the inertia tensor. Perception \& Psychophysics, 58, 1191 1202.

Pagano, C. C., Fitzpatrick, P., \& Turvey, M. T. (1993). Tensorial basis to the constancy of perceived object extent over variations of dynamic touch. Perception \& Psychophysics, 54, 4345.

Pagano, C. C., Garrett, S., \& Turvey, M. T. (1996). Is limb proprioception a function of the limbs' inertial eigenvectors. Ecological Psychology, 8, 43-69.

Pagano, C. C., Kinsella-Shaw, J., Cassidy, P., \& Turvey, M. T. (1994). Role of the inertia tensor in perceiving where an object is grasped. Journal of Experimental Psychology: Human Perception and Performance, 20, 276-285.

Pagano, C. C. \& Turvey, M. T. (1992). Eigenvectors of the inertia tensor and perceiving the orientation of a hand-held object by dynamic touch. Perception \& Psychophysics, 52, 617-624.

Pagano, C. C., \& Turvey, M. T. (1995). The inertia tensor as a basis for the perception of limb orientation. Journal of Experimental Psychology: Human Perception and Performance, 21, 1070-1087.

Peck, A. J., Jeffers, R. G., Carello, C., \& Turvey, M. T. (1996). Haptically perceiving the length of one rod by means of another. Ecological Psychology, 8, 237-258.

Pellionisz, A., \& Llinás, R. (1985). Tensor network theory of the metaorganization of functional geometries in the central nervous sytem. Neuroscience, 16, 245-273.

Rosenblum, L. D., Wuestefeld, A. P., \& Anderson, K. L. (1996). Auditory reachability: An affordance approach to the perception of sound source distance. Ecological Psychology, $8,1-24$.

Shaw, R. E., \& Bransford, J. D. (1977). In R. E. Shaw \& J. D. Bransford (Eds.), Perceiving, acting and knowing (pp. 1-39). Hillsdale, NJ: Erlbaum.

Shaw, R. E., \& Kinsella-Shaw, J. M. (1988). Ecological mechanics: A physical geometry for intentional constraints. Human Movement Science, 7, 155-200.

Shockley, K., Carello, C., \& Turvey, M. T. (under review). Affordances and the physical basis of perceived heaviness.

Solomon, H. Y. (1988). Movement-produced invariants in haptic explorations: An example of a self-organizing, information-driven, intentional system. Human Movement Science, 7, 201223.

Solomon, H. Y., \& Turvey, M. T. (1988). Haptically perceiving the distances reachable with handheld objects. Journal of Experimental Psychology: Human Perception and Performance, 14, 404-427.

Solomon, H. Y., Turvey, M. T., \& Burton, G. (1989). Perceiving extents of rods by wielding: Haptic diagonalization and decomposition of the inertia tensor. Journal of Experimental Psychology: Human Perception and Performance, 15, 58-68.

Stevens, J. C., \& Rubin, L. L. (1970). Psychophysical scales of apparent heaviness and the sizeweight illusion. Perception \& Psychophysics, $\underline{8}, 225-230$.

Turvey, M. T. (1996). Dynamic touch. American Psychologist, 51, 1134-1152.

Turvey, M. T., Burton, G., Amazeen, E. L., Butwill, M., \& Carello, C. (1998). Perceiving the width and height of a hand-held object by dynamic touch. Journal of Experimental Psychology: Human Perception and Performance, 24, 35-48.

Turvey, M. T., Burton, G., Pagano, C. C., Solomon, H. Y., \& Runeson, S. (1992). Role of the inertia tensor in perceiving object orientation by dynamic touch. Psychology: Human Perception and Performance, 3 , 714-727.

Turvey, M. T., \& Carello, C. (1981). Cognition: The view from ecological realism. Cognition, 10 , 313-321.

Turvey, M. T., \& Carello, C. (1995a). Dynamic touch. In W. Epstein \& S. Rogers (Eds.), Handbook of perception and cognition, Vol. V. Perception of space and motion (pp. 401490). San Diego: Academic Press. 
Turvey, M. T., \& Carello, C. (1995b). Some dynamical themes in perception and action. In R. Port $\&$ T. van Gelder (Eds.), Mind in motion. Cambridge, MA: MIT press.

Turvey, M. T., Carello, C., Fitzpatrick, P., Pagano, C. C., \& Kadar, E. (1996). Spinors and selective dynamic touch. Journal of Experimental Psychology: Human Perception and Performance, 22, 1113-1126.

Turvey, M. T., Carello, C., \& Kim, N-G. (1990). Links between active perception and the control of action. In H. Haken \& M. Stadler (Eds.), Synergetics of cognition (pp. 269-295). Berlin: Springer Verlag.

Turvey, M. T., Park, H-S., Dumais, S. P., \& Carello, C. (1998). Nonvisible perception of segments of a handheld object and the attitude spinor. Journal of Motor Behavior, 30, 3-19.

Turvey, M. T., \& Shaw, R. E. (1995). Toward an ecological physics and a physical psychology. In R. Solso \& D. Massaro (Eds.), The science of the mind: 2001 and beyond. Oxford: Oxford University Press.

Turvey, M. T., Shaw, R. E., Reed, E. S., \& Mace, W. M. (1981). Ecological laws of perceiving and acting: In reply to Fodor and Pylyshyn (1981). Cognition, 9, 237-304.

Vaught, Simpson, \& Ryder, (1968).

Weber, E. H. (1978). The sense of touch (H. E. Ross Ed. and Translator). London: Academic Press. (Original work published 1834). 


\section{Footnotes}

1The precise cause is not known. It is thought that there may be some connection to the body's reaction to a viral infection in which cells produced to attack the foreign virus attack the peripheral nerves as well. Partial neuropathies are more prevalent, accompanying a variety of conditions including, most commonly, diabetes.

2Indeed, there is no treatment for his condition in terms of restoring lost feeling. What physical therapy allowed Mr. Waterman, instead, was to discover for himself the tricks and strategies that he could use to accomplish his old activities in new, non-haptic ways.

${ }^{3}$ This is so even if the movements do not seem overtly rotational. To appreciate this, draw the outline of a large box in the air in front of you and pay attention to what is happening at the wrist, elbow, and shoulder joints. Even as the motion of the endpoint is linear, that motion is accomplished by cascaded rotations of hand about wrist, forearm about elbow, and whole arm about shoulder.

${ }^{4}$ This is, of course, the rationale behind the use of ankle weights in exercise. Because the weighted leg is harder to move, more effort is required, and more calories are burned with the more strenuous exercise.

5 A tensorial treatment of the muscle sense has been promoted by Pellionisz and Llinás (1985) and Solomon (1988).

${ }^{6}$ The question of error in perception is neither trivial nor straightforward. Elsewhere, we have argued that applying the label error to perception only makes sense in a theory that describes perception as a process of making propositions about the environment. In a theory that characterizes perception as resonating to available structure, that process can no more be wrong than any other process of physical equilibration. Thorough discussions of the possibility of error in perception can be found elsewhere (e.g., Carello \& Turvey, 1985; Michaels \& Carello, 1981; Turvey, Shaw, \& Mace, 1982).

${ }^{7}$ For perceiving properties of objects in the hand, the physical constraint is the inertia tensor calculated about a center of rotation in the wrist even when movement is permitted about other joints of the arm (Pagano, Fitzpatrick, \& Turvey, 1993). We were, in fact, somewhat hesitant to discover this important fact, fearing that free limb movement might require us to confront time-varying tensors. But as it happens, when an object is grasped firmly in the hand, the wrist is the only point that remains a fixed distance from the object during wielding. The linked-cylinder design of the arm allows variability and flexibility in movement dynamics while preserving an anchor for invariant spatial perception.

${ }^{8}$ It should be noted that, unlike other examples of probing, calculations of $\mathrm{I}, \underline{\mathrm{CP}}$, and so on, for aperture size require the hand as well as the probe. The reason for this difference is not understood.

${ }^{9}$ Although we have not discussed it here, a number of experiments over the years have examined perception of object properties by static holding or, more accurately, minimal movement. Whole length and partial length can both be perceived successfully under instructions to hold rather than wield the rods (Burton \& Turvey, 1990; Carello et al., 1996). If the participants were truly able to hold the rods still, then the resistance to rotational inertia would not available to deform the tissues of the hand and arm. And while it is the case that static moment tends to dominate moment of inertia as the major constraint on perceived whole length (Burton \& Turvey, 1990), the inertia tensor is available nonetheless. Our suspicion is that the minimal movements accompanying respiration, cardiac pulse, and muscular tremor are sufficient to promote this availability (Carello et al., 1996). 